\title{
Electric Utility Restructuring and the \\ California Biomass Electricity Industry
}

Future Resources Associates, Inc.

Berkeley, California

Technical Monitor: Ralph Overend

National Renewable Energy Laboratory

1617 Cole Boulevard

Golden, Colorado 80401-3393

A national laboratory of the U.S. Department of Energy

Operated by Midwest Research Institute

for the U.S. Department of Energy

Under Contract No. DE-AC36-83CH10093

Prepared under Subcontract Number ACK-6-16357

May 1997 


\section{NOTICE}

This report was prepared as an account of work sponsored by an agency of the United States government. Neither the United States government nor any agency thereof, nor any of their employees, makes any warranty, express or implied, or assumes any legal liability or responsibility for the accuracy, completeness, or usefulness of any information, apparatus, product, or process disclosed, or represents that its use would not infringe privately owned rights. Reference herein to any specific commercial product, process, or service by trade name, trademark, manufacturer, or otherwise does not necessarily constitute or imply its endorsement, recommendation, or favoring by the United States govemment or any agency thereof. The views and opinions of authors expressed herein do not necessarily state or reflect those of the United States government or any agency thereof.

Available to DOE and DOE contractors from:

Office of Scientific and Technical information (OSTI)

P.O. Box 62

Oak Ridge, TN 37831

Prices available by calling (423) 576-8401

Available to the public from:

National Technical Information Service (NTIS)

U.S. Department of Commerce

5285 Port Royal Road

Springfield, VA 22161

(703) $487-4650$ 


\section{DISCLAIMER}

Portions of this document may be illegible in electronic image products. Images are produced from the best available original document. 


\section{Contents}

Page

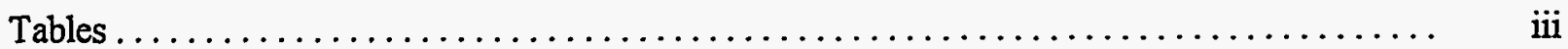

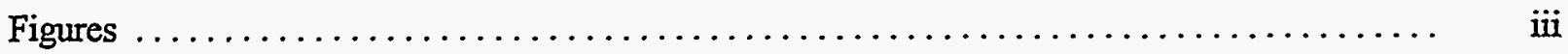

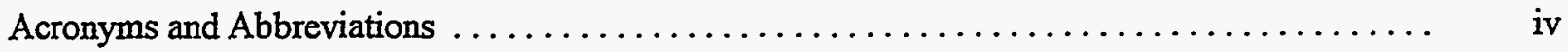

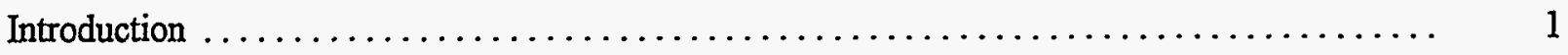

The Evolving Regulatory Environment for Biomass Energy $\ldots \ldots \ldots \ldots \ldots \ldots \ldots \ldots \ldots .2$

Proposals to the California Renewables Working Group $\ldots \ldots \ldots \ldots \ldots \ldots \ldots \ldots \ldots \ldots$

Renewables Program Implementation Proposals ........................ 5

Positions of the Proposals with Respect to Key Issues $\ldots \ldots \ldots \ldots \ldots \ldots \ldots \ldots \ldots \ldots \ldots$

Areas of Commonality and Difference among the Proposals ................. 25

Fiscal Tools and Technology Investments in Biomass Power $\ldots \ldots \ldots \ldots \ldots \ldots \ldots \ldots \ldots$



\section{Tables}

Table 1. Proposals to the CPUC Renewables Working Group $\ldots \ldots \ldots \ldots \ldots \ldots \ldots$

Table 2. Features of Proposals to Implement the CPUC Renewables Policy .......... 9

Table 3. Cost of Electricity Production from Biomass $\ldots \ldots \ldots \ldots \ldots \ldots \ldots \ldots \ldots \ldots$

\section{Figures}

Figure 1. Timeline for Electricity Restructuring in California $\ldots \ldots \ldots \ldots \ldots \ldots \ldots .4$

Figure 2. Classification of Program Proposals $\ldots \ldots \ldots \ldots \ldots \ldots \ldots \ldots \ldots \ldots \ldots$

Figure 3. Cost of Biomass Power Production $\ldots \ldots \ldots \ldots \ldots \ldots \ldots \ldots \ldots \ldots \ldots$ 


\section{Acronyms and Abbreviations}

\begin{tabular}{|c|c|}
\hline AB 1890 & California's Restructuring Law, signed September 24, 1996 \\
\hline bdt & bone-dry ton equivalents of biomass fiber, the basis of fuel pricing in California \\
\hline Cal/EPA & California Environmental Protection Agency \\
\hline CBEA & California Biomass Energy Alliance \\
\hline CEC & California Energy Commission \\
\hline CPUC & California Public Utilities Commission \\
\hline CTC & competitive transition charge, the name given to stranded costs in California \\
\hline EPAct & the federal Energy Policy Act of 1992 \\
\hline ISO & independent system operator \\
\hline MRPR & minimum renewables purchase requirement \\
\hline O\&M & operations and maintenance \\
\hline PBR & performance-based ratemaking \\
\hline PG\&E & Pacific Gas and Electric Corporation \\
\hline PPA & power purchase agreement \\
\hline REPI & renewable energy production incentive \\
\hline PURPA & Public Utility Regulatory Act of 1978 (federal) \\
\hline RD\&D & research, development, and demonstration \\
\hline PV & photovoltaics \\
\hline QF & qualifying facility, a designation regulated by the FERC \\
\hline RPS & renewable portfolio standard \\
\hline SCE & Southern California Edison Company \\
\hline SRAC & short-run avoided cost, the basis of energy payments to qualifying facilities in California \\
\hline T\&D & transmission and distribution \\
\hline UDC & utility distribution company \\
\hline
\end{tabular}




\section{Introduction}

A shock jolted the electric power industry in April 1994, when the California Public Utilities Commission (CPUC) announced its intention to "restructure" the industry. The proposal, commonly referred to as retail wheeling, is based on the principle that market deregulation and competition will bring down the cost of electricity for all classes of customers. It would effectively break up the monopoly status of the regulated utilities and allow customers to purchase electricity directly from competing suppliers. According to the original CPUC proposal, cost alone would be the basis for determining which generating resources would be used. The proposal was modified in response to public inputs, and issued as a decision at the end of 1995. The final proposal recognized the importance of renewables, and included provisions for a minimum renewables purchase requirement (MRPR). A Renewables Working Group convened to develop detailed proposals for implementing the CPUC's renewables program. Numerous proposals, which represented the range of possible programs that can be used to support renewables within the context of a restructured electric utility industry, were received.

Renewable energy generators provide important benefits to the state's power system that are not reflected in the short-run avoided cost (SRAC) rate that is the basis for the payment of electricity revenues* to renewable power producers under current contracts with the electric utility companies. These benefits include environmental advantages, rural employment and economic development opportunities, and resource diversity and distributional benefits. Renewables generally have lower levels of environmental impact than fossil fuel power generating systems. However, biomass power systems have the unique advantage, in addition to avoiding fossil fuel use, of providing ancillary environmental services by offering a beneficial use alternative, which is environmentally superior to conventional waste disposal practices, for a variety of waste materials. The loss of these services would have severe negative environmental consequences, and would complicate California's efforts to comply with air quality and solid waste diversion requirements, and to implement fire and water plans.

The California renewable energy industries have worked diligently during the past couple of years to achieve public policies conducive to the future of renewable energy production within the context of electric market restructuring and the evolving competitive electric services industry. The biomass power industry has organized itself as the California Biomass Energy Alliance (CBEA), and has participated vigorously in the regulatory and legislative processes. In order to reward biomass power generators for the special services they provide, CBEA has promoted the concept of providing incentives specifically targeted to biomass within the context of any renewables program enacted in the state. This concept has been embraced by the other renewables industry organizations, but resisted by the utilities.

A major part of this study involved an effort to characterize and analyze some proposals that have been made to the CPUC Renewables Working Group for implementing the renewables program included in the CPUC's December 20, 1995, restructuring decision. $A B$ 1890, California's landmark restructuring legislation, was signed into law on September 24, 1996 and superseded the CPUC restructuring proposal. Nevertheless, the proposals to the Renewables Working Group provide a broad perspective on the design of renewables programs within a restructured market, and the analysis is relevant to several jurisdictions. The report begins with a descriptive history of the restructuring process in California. This is followed by an analysis of proposals made to the CPUC for implementing the commission's restructuring decision. The report ends with a consideration of the future prospects for the industry in the context of a restructured electric utility market. 


\section{The Evolving Regulatory Environment for Biomass Energy}

Two major factors that contributed to the development of the biomass power industry in California during the 1980s were a stable regulatory environment and the availability of attractive long-term power purchase agreements (PPAs). Utilities were regulated monopolies, and were expected to remain so. The Public Utility Regulatory Policy Act (PURPA) of 1978 required the electric utility companies to purchase power from independent generating sources and give special status to renewables. The PPAs during the mid-1980s offered long-term fixed pricing provisions for energy and capacity, an important factor for developers to procure funding for their projects.

By the early 1990s natural gas prices had stabilized at a low level, and electric generating capacity in the western United States exceeded demand. The California biomass power industry had matured and was operating in a stable mode. However, the regulatory environment embarked on a process of accelerating change in 1992 that will redefine electricity markets throughout the country by early in the next century. The process began with the passage of the federal Energy Policy Act of 1992 (EPAct), which, among its many provisions, provided for open access on the nation's interstate electricity transmission networks. California took the lead in deregulating the electric utility industry in April 1994, when the CPUC issued the Blue Book proposal to restructure the electric utility industry. This proposal launched the electric utility deregulation movement in the United States. The future electricity market would feature a competitive power generation sector, and a new class of independent energy services providers would have direct access to the grid and be able to sell power directly to customers. Full price competition would favor the lowest-cost generators, which in California would include mainly hydroelecric, outof-state coal, and efficient gas-fired generators. Biomass power clearly has a higher cost of generation than these sources. Figure 1 shows a timeline of the evolving restructuring movement.

In response to the Blue Book proposal the biomass power producers of California, as well as other renewable energy producers, began to organize into groups to represent their interests in regulatory and legislative forums. The biomass power producers formed the CBEA, which eventually attracted the participation of more than $90 \%$ of the biomass power generating capacity in the state. The renewables organizations, in cooperation with environmental groups, worked hard to achieve recognition for the economic, diversity, and environmental benefits of renewable generating sources within the context of electric utility restructuring.

The original Blue Book proposal provided for competition among generating sources on the basis of price alone, without regard to environmental impacts, source diversity, or regional economic development. The second version of the proposal, issued in March 1995, included an explicit recognition of the importance of maintaining environmental and diversity considerations in selecting generating sources. The CPUC's third-generation restructuring proposal, adopted in December 1995, called for the establishment of an MRPR for all electric services providers under the jurisdiction of the CPUC. The MRPR, commonly known as the renewable portfolio standard (RPS), was intended to preserve the renewable energy industry and promote growth in renewables. These were acknowledged to be state-endorsed, public purpose goals.

In early 1996, a Renewables Working Group was formed under the auspices of the CPUC to develop policy strategies for implementating the CPUC's renewables policy. The group had the participation of the renewable power industries, the major private and public electric utility companies, state agencies, and consumer and environmental advocacy groups. Six comprehensive implementation proposals, and two adjunct proposals, were developed by working group members and submitted to the group for consideration. The group prepared a report to the CPUC that presented the proposals and analyzed their commonalities and differences (see the section, Analysis of Proposals to the Califomia Renewables Working Group).

In parallel action the CBEA introduced legislation that would establish a minimum purchase requirement for biomass power, and laid the groundwork for a broader-based RPS program. The bill, AB 1202, was introduced 
originally during the 1995 legislation session, where it gained committee passage but was not considered by the full Assembly. It was reintroduced in the 1996 session and passed the Assembly in the spring of 1996. AB 1202 was passed on to the Senate, along with several other bills related to electricity policy and deregulation. All restructuring matters were sent to a joint Assembly-Senate conference committee, which worked from a blank slate to craft electric utility deregulation.

The California Electric Industry Restructuring Legislative Conference Committee began to deliberate in mid-July, facing an absolute deadline of August 31 for the 1996 legislative session. In the overall scheme the specific issues that related to the renewable energy industry constituted a small, but significant, component. The initial positions voiced by participating parties spanned the spectrum from requests for increased support for renewables, to no support at all. The renewable energy industries advocated the MRPR approach, consistent with the CPUC's ongoing restructuring program, and with $\mathrm{AB} 1202$.

Early in the committee's deliberations the MRPR was declared a dead option. Only a public goods surchargefunded program would be considered to support renewable energy. The industry was allocated approximately $\$ 500$ million, to be made available during a 4 - to $4 \frac{1}{2}$-year period (the transition period to the full implementation of restructuring). No mandated renewables program was included for the post-transition market. The California Energy Commission (CEC) was charged with recommending how to allocate and distribute the renewable energy funds, with the caveat that at least of $40 \%$ of the funds must be allocated to new renewables, and at least $40 \%$ to current renewables.

The electric utility restructuring legislation crafted by the committee, $\mathrm{AB} 1890$, was signed into law on September 24, 1996. $\mathrm{AB} 1890$ makes special reference to biomass as an important generating source for California. In addition to eligibility for renewables transition funding, $\mathrm{AB} 1890$ directs the California Environmental Protection Agency (Cal/EPA) to report to the legislature on the benefits associated with the biomass power industry, and to suggest cost-shifting strategies that would transfer some costs of biomass power generation away from the electric ratepayer and onto the beneficiaries of the environmental services provided by biomass generation. Shifting approximately the cost of fuel procurement away from the electric ratepayer would allow the biomass power industry to be competitive in the restructured electricity market.

Determining the level of funding that would be required to preserve the renewable energy industry in California is possible without knowing the market price for energy in the deregulated market, but most observers believe that the legislation produced by the conference committee fails to provide enough support to preserve the industry through the transition period. Furthermore, the legislation provides no specific support for renewables in the long term. There will almost certainly be a shrinkage of renewable energy generation if the legislature's restructuring program is not modified. Further restructuring legislation surely will be forthcoming during the next legislative session, as many issues remain unresolved. 
Figure 1

\section{Timeline for Electricity Restructuring in California}

\begin{tabular}{|c|c|c|c|c|c|c|}
\hline & 1992 & 1993 & 1994 & 1995 & 1996 & 1997 \\
\hline \multicolumn{7}{|c|}{ Cal. Public Utilities Commission } \\
\hline EPAct & $\diamond$ & & & & & \\
\hline Blue Book Proposal & & & $\diamond$ & & & \\
\hline Restructuring Working Group & & & $\Delta$ & & & \\
\hline CPUC Split Decision & & & & $\diamond$ & & \\
\hline Edison MOU & & & & $\Delta \varpi \nabla$ & & \\
\hline CPUC Decision, Roadmap & & & \multicolumn{4}{|c|}{$\bullet$} \\
\hline Renewables Working Group & & & & \multicolumn{3}{|c|}{$\Delta \Delta_{\cos } \nabla$} \\
\hline \multicolumn{7}{|l|}{ Cal._Legislature } \\
\hline$A B 1202$ & & & \multicolumn{4}{|c|}{$O m$} \\
\hline$A B 1890$ & & & & & $\diamond$ & \\
\hline CEC Report to Legislature & & & & & & \\
\hline CaVIEPA Report to Legislature & & & & & & $\nabla$ \\
\hline
\end{tabular}

EPAct: Energy Policy Act, 1992 (fed). Mandated open access on interstate power transmission systems.

Blue Book Proposal: Original restructuring proposal of the CPUC.

Restructuring Working Group: Working group formed to respond to the CPUC about the Blue Book. CPUC Split Decision: Second-generation CPUC restructuring proposal, which was a split decision. Edison MOU: Several major players, led by SCE, negotiated an MOU re. restructuring principles. CPUC Decision, Roadmap: Third CPUC restructuring decision, followed up by roadmap on implementation. Renewables Working Group: Group formed to propose implementation strategies for renewables program. AB 1202: CBEA-supported bill to create an RPS. Passed Assembly, never considered by Senate. $A B$ 1890: Landmark Restructuring Legislation signed into law, September 24, 1996. CEC Report to Legislature: Report on allocation of transition funds for renewables. Cal/EPA Report to Legislature: Report on cost-shifting strategies for the biomass power industry. 


\section{Proposals to the California Renewables Working Group}

This section is taken from the Renewables Working Group report to the CPUC on implementing a renewables program within the context of electric utility restructuring. This material appears in the Working Group Report as the chapter titled: "Commonalities and Differences among Proposals." The California Renewables Working Group met biweekly from February through August 1996. From the beginning, the group acknowledged that no single approach to developing a renewables policy to implement the commission's restructuring decision would be agreed upon by all participants. The group invited all interested parties to submit comprehensive prog-ram proposals for implementing the CPUC's renewable energy policy. It specifically requested comprehensive program proposals to avoid limited-purpose proposals that addressed various pieces of a renewables program, but no way to understand how the pieces would fit together into an integrated, total program. The group received six comprehensive program proposals. Five presented strategies for implementating a program based on the MRPR approach. The sixth is for a surcharge-funded program that distributes renewable production credits on the basis of a competitive bidding process. The group also received two adjunct proposals that seek to support specific types of technologies within the context of whichever overall renewables program is adopted. The eight proposals provide a variety of approaches to the development of a workable renewables policy for California, and illustrate the range of issues that must be addressed in formulating the program.

\section{Renewables Program Implementation Proposals}

There are several ways to separate the proposals into functional categories to compare and contrast them. This can be done in a hierarchical structure, as illustrated in Figure 2. The names of the proposals and their sponsors are shown in Table 1. The first category concerns whether the proposed program is based on establishing an MRPR. The next is based on the unit of measurement used, which can be either energy units (kWh) or capacity units $(\mathrm{kW})$. The third differentiates between proposals that do or do not include specified technology bands to promote targeted technologies. The fourth addresses the issue of whether hydroelectric generating systems are included in the program. The final category concerns the issues of program enforcement, penalties, and cost control. This structure allows all six comprehensive program proposals to be differentiated with respect to their most significant functional differences. The adjunct proposals are also included in the figure.

A summary of the proposals and some of their distinguishing characteristics follows:

\section{Comprehensive Program Proposals}

Renewables industry organizations: Includes an MRPR, is based on energy units, has one specified technology band for biomass, excludes hydrogen, employs a high, punitive penalty intended to motivate full compliance, and uses a credit price cap to control program costs.

IEP: Includes an MRPR, is based on energy units, has one specified technology band for biomass, excludes hydrogen, and is predicated on voluntary compliance through green marketing by electricity providers, with a requirement for utility distribution companies (UDCs) to purchase the necessary quantity of additional renewables to meet the MRPR standard, which will be enforced by performance-based ratemaking (PBR) incentives.

NCPA: Includes an MRPR, is based on capacity units, has no specified technology bands, includes hydrogen, and employs a penalty, intended to motivate full compliance, that applies to all kilowatt-hours sold by a noncomplying electric services provider.

SCE/PG\&E: Includes an MRPR, is based on energy units, has no specified technology bands, excludes hydrogen, provides for enforcement penalties to be set by the program administrator, and uses a credit price cap to control program costs. 


\section{Table 1}

\section{Sponsors of Proposals to the Renewables Working Group}

Renewables Industry Organizations: American Wind Energy Assoc., California Biomass Energy Alliance, Geothermal Energy Assoc., Solar. Thermal Energy Assoc., Union of Concerned Scientists, California Integrated Waste Management Board.

IEP: Independent Energy Producers Assoc.

NCPA: Northern California Power Agency

SCE/PG\&E: Southern California Edison Co., Pacific Gas and Electric Co.

SMUD: Sacramento Municipal Utility District.

EDF et. al.: Environmental Defense Fund, Cambrian Energy, Genesis Co., Laidlaw Inc., Los Angeles Sanitation District, Neo Corp., Orange and Sonoma Counties, City of Sacramento.

BWG: Biogas Working Group.

CalSEIA et. al.: California Solar Energy Industries Assoc., Solar Energy Industries Assoc., Energy Technologies Development Division of the California Energy Commission, Natural Resources Defense Council, Sacramento Municipal Utility District. 
Figure 2: Classification of Program Proposals

1. Based on MRPR Standard?

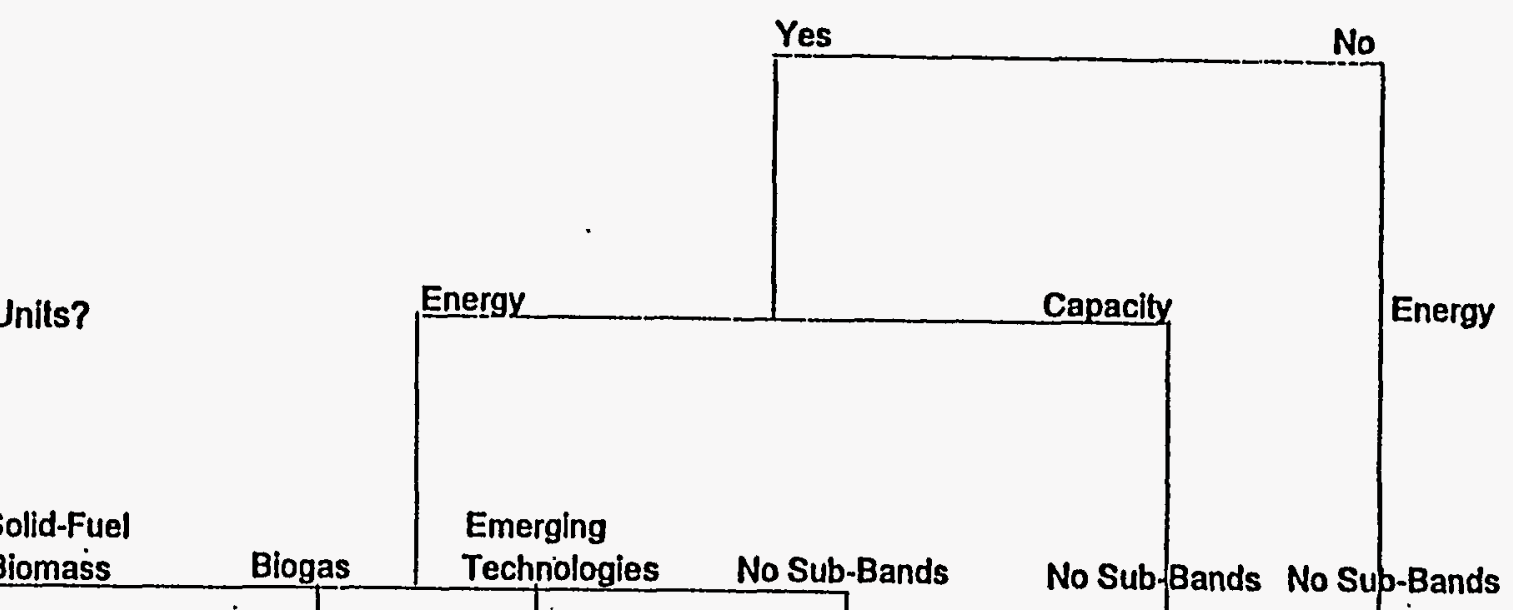

3. Specified Technology Bands?

Biomass Biogas Technologies

No Sub-Bands

2. Denominated in Energy or Capacity Units?

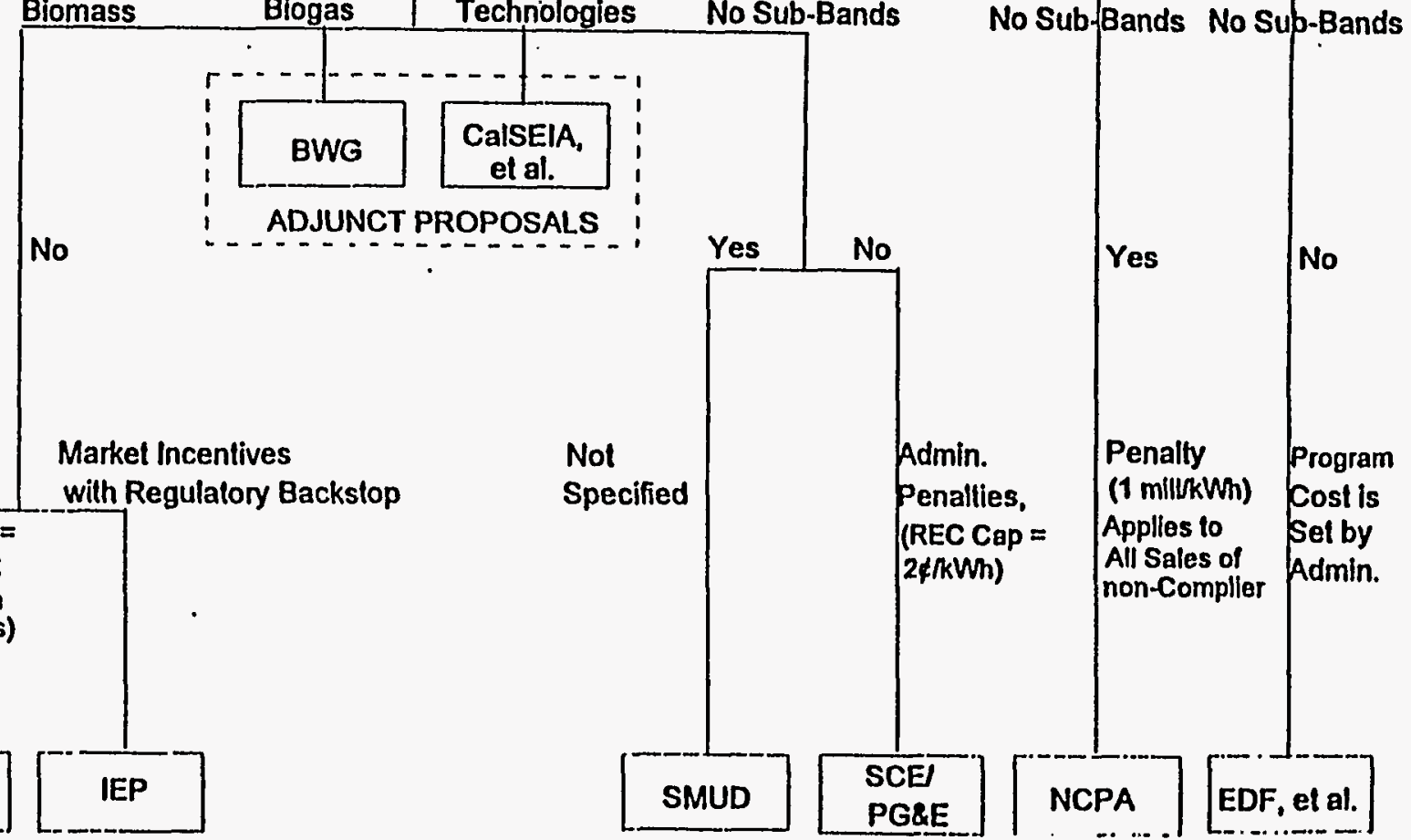

Nole: Adjunct proposals can be effached to any of the comprehensive propram proposals 
SMUD: Includes an MRPR, is based on energy units, has no specified technology bands, includes hydrogen, and does not address the issues of enforcement, penalties, or program cost.

EDF et. al: Based on a surcharge funding approach, uses credits based on energy units, has no specified technology bands, excludes hydrogen, and provides for enforcement by the program administrator, with program cost set administratively.

\section{Adjunct Proposals}

The adjunct proposals are limited-purpose proposals that target emerging renewable energy technologies. These technologies are not yet fully competitive with conventional renewable generation, but the proposers believe they improve environmental quality or increase resource diversity, or both. They can be applied to any comprehensive program proposal presented in this report.

BWG: Proposes to create special-purpose "greenhouse environmental credits" equal in value to a renewable energy credit for promoting the growth of electricity generation from landfill gas and other biogas sources, technologies that help mitigate the effects of methane gas emissions.

CaISEIA et. al.: Proposes to create small markets for emerging technologies, such as photovoltaics (PV), that are progressing from the RD\&D phase toward full market competitiveness with more established generating technologies.

\section{Positions of the Proposals with Respect to Key Issues}

The six full program proposals and two adjunct proposals offer a wide range of options regarding the structure and design of an effective renewable energy program. Table 2 presents the major issues that should be a part of any renewables program developed by the CPUC or the California State Legislature, and summarizes the positions of the proposals with respect to each issue. For the two adjunct proposals the table shows entries only for those categories addressed specifically by the proposals. The table illustrates the range of approaches proposed for dealing with key issues identified by the CPUC and the Working Group. These issues are analyzed below.

\section{Program Obligation Issues}

Basis for the Obligation. The CPUC restructuring decision recommends that an MRPR be established "to meet our resource diversity goals" (p. 150, D95-12-063 as modified by D.96-01-009). The decision further calls for establishing an effective enforcement mechanism to ensure compliance with the program. Each comprehensive program proposal offers a distinct approach to creating and enforcing a renewables program to fulfill the CPUC's policy objectives for renewables. Five of the six present strategies to implement the MRPR mechanism incorporated in the CPUC restructuring decision. The EDF et al. proposal employs an alternative approach to achieve the CPUC's policy objectives, in which a program for new renewables would be funded by a surcharge on electricity bills, with surcharge funds distributed to new renewable energy projects as production credits on the basis of a competitive bidding program. All the MRPR-based proposals include the use of tradable renewable energy credits (RECs) to facilitate compliance and spread the costs of the program equitably across the state. Programs based on the MRPR mechanism aim to achieve a predictable quantity of renewable energy production, and rely on market competition to minimize program cost. The surcharge-funded production credit approach ensures a predictable program cost, with competition for surcharge funds used to maximize the quantity of renewables generated. 
Table 2: Features of Proposals to Implement The CPUC Renewables Policy

1. Program Obllgatlon

\begin{tabular}{|c|c|c|c|c|c|c|c|}
\hline Proposal & $\begin{array}{l}\text { Program } \\
\text { Based on } \\
\end{array}$ & $\begin{array}{c}\text { Basis for } \\
\text { Initlal MRPR }\end{array}$ & $\begin{array}{l}\text { Inillal MRPR with Full } \\
\text { Implementation }\end{array}$ & $\begin{array}{c}\text { Increase In MRPR } \\
1999-2000\end{array}$ & $\begin{array}{l}\text { MRPR with CPUC } \\
\text { Implementation }\end{array}$ & $\begin{array}{c}\text { MRPR } \\
\text { Applled to }\end{array}$ & $\begin{array}{c}\text { Specifled } \\
\text { Technology Bands }\end{array}$ \\
\hline \multicolumn{8}{|c|}{ Comprehonslve Program Proposals } \\
\hline \multicolumn{8}{|l|}{ MRPRProposals } \\
\hline AWEA et. al. & energy & $90 \%$ of 1999 level & $11.6 \%$ & $+0.2 \% / y$ & adjust upward & energy sales to users & Biomass \\
\hline IEP & energy & 1998 leval + BRPU & not available & None & CPUC only needed & energy sales to users & Biomass \\
\hline NCPA & capacity & ER '94 proj. for 1998 & $18.0 \%$ & None & state-wide only & ave. monthly poak cap. & None \\
\hline SCE/PG\&E & energy & approx. lavel of 1990 s & $10.0 \%$ & None & same as slate-wide & energy sales to users & None \\
\hline SMUD & energy & 1994 level & $21.0 \%$ & None & state-wide only & energy sales to users & None \\
\hline \multicolumn{8}{|l|}{ Surcharge Proposal } \\
\hline EDF ot. al. & energy & NA & NA & NA & NA & NA & None \\
\hline \multicolumn{8}{|l|}{ Adjunct Proposals } \\
\hline $\begin{array}{l}\text { Biogas Working Group } \\
\text { CalSEIA ot. al. }\end{array}$ & $\begin{array}{l}\text { energy } \\
\text { energy }\end{array}$ & $\begin{array}{l}\text { not addressed } \\
\text { not addressed }\end{array}$ & $\begin{array}{l}\text { not addressed } \\
\text { not addressed }\end{array}$ & $\begin{array}{l}\text { not addressed } \\
100 \mathrm{GWh} / \mathrm{yr}\end{array}$ & $\begin{array}{l}\text { not addressed } \\
\text { not addressed }\end{array}$ & $\begin{array}{l}\text { not addressed } \\
\text { not addressed }\end{array}$ & $\begin{array}{l}\text { Biogas } \\
\text { Emerging Technologies }\end{array}$ \\
\hline
\end{tabular}

\section{Program Ellglbility}

\begin{tabular}{|c|c|c|c|c|c|c|c|}
\hline Proposal & $\begin{array}{c}\text { Hydro } \\
\text { Eligibllity }\end{array}$ & $\begin{array}{l}\text { Ellglbility of Non- } \\
\text { Callf. Renewables }\end{array}$ & $\begin{array}{l}\text { Eligibllity of Bulk } \\
\text { Utility Renewables }\end{array}$ & $\begin{array}{l}\text { Ellglbility of UDC } \\
\text { Dist. Renewables }\end{array}$ & $\begin{array}{l}\text { Eliglbility of } \\
\text { Existing aFs }\end{array}$ & $\begin{array}{l}\text { Eliglbillity of Power } \\
\text { Gen. for On-Site Use }\end{array}$ & $\begin{array}{l}\text { Eligibility } \\
\text { of Hybrids }\end{array}$ \\
\hline \multicolumn{8}{|c|}{ Comprehensive Program Proposals } \\
\hline \multicolumn{8}{|l|}{ MRPR Proposals } \\
\hline $\begin{array}{l}\text { AWEA el. al. } \\
\text { IEP } \\
\text { NCPA } \\
\text { SCE/PG\&E } \\
\text { SMUD }\end{array}$ & $\begin{array}{c}\text { not eligible } \\
\text { small hydro eligible } \\
\text { eligible } \\
\text { not eligible } \\
\text { eligible }\end{array}$ & $\begin{array}{c}\text { BECs Calit, only } \\
\text { eligible } \\
\text { nol eligible } \\
\text { eligible } \\
\text { eligible }\end{array}$ & $\begin{array}{l}\text { eligible } \\
\text { eligible } \\
\text { eligible } \\
\text { eligible } \\
\text { eligible }\end{array}$ & $\begin{array}{l}\text { needs resolution } \\
\text { needs resolution } \\
\text { oligible } \\
\text { needs resolution } \\
\text { nol addressed }\end{array}$ & $\begin{array}{l}\text { eligible } \\
\text { eligible } \\
\text { eligible } \\
\text { eligible } \\
\text { eligible }\end{array}$ & $\begin{array}{l}\text { not eligible } \\
\text { eligible } \\
\text { not eligible } \\
\text { not eligible } \\
\text { eligible }\end{array}$ & $\begin{array}{l}\text { RECs il qualify } \\
\text { RECs il qualify } \\
\text { RCCs il qualily } \\
\text { pro-rate RECs } \\
\text { pro-rate RECs }\end{array}$ \\
\hline $\begin{array}{l}\text { Surcharge Proposal } \\
\text { EDF et. al. }\end{array}$ & \multicolumn{6}{|c|}{ Surcharge Proposal } & pro-rate credils \\
\hline \multicolumn{8}{|l|}{ Adjunct Proposals } \\
\hline $\begin{array}{l}\text { Biogas Working Group } \\
\text { CalSEIA ot. al. }\end{array}$ & $\begin{array}{l}\text { not eligible } \\
\text { not eligible }\end{array}$ & $\begin{array}{l}\text { eligible } \\
\text { eligible }\end{array}$ & $\begin{array}{l}\text { eligible } \\
\text { eligible }\end{array}$ & $\begin{array}{l}\text { not addressed } \\
\text { neads resolution }\end{array}$ & $\begin{array}{c}\text { eligible } \\
\text { not eligible }\end{array}$ & $\begin{array}{l}\text { not eligible } \\
\text { grid connected elig. }\end{array}$ & $\begin{array}{l}\text { prorate RECs } \\
\text { RECs il qualify }\end{array}$ \\
\hline
\end{tabular}




\section{Table 2: Features of Proposals to Implement The CPUC Renewables Policy (cont.)}

3. Program Administration

\begin{tabular}{|c|c|c|c|c|c|c|c|}
\hline Proposal & $\begin{array}{l}\text { Application } \\
\text { of Program }\end{array}$ & $\begin{array}{l}\text { Implementatlon in } \\
\text { Phases Permitted }\end{array}$ & $\begin{array}{c}\text { Administrator } \\
\text { (full Implementatlon) }\end{array}$ & $\begin{array}{l}\text { Funding } \\
\text { Mechanism }\end{array}$ & $\begin{array}{l}\text { Program } \\
\text { Lifetime }\end{array}$ & $\begin{array}{l}\text { Perlod to Determine } \\
\text { Compllance }\end{array}$ & $\begin{array}{c}\text { Commerclallzatlon } \\
\text { Support }\end{array}$ \\
\hline \multicolumn{8}{|c|}{ Comprehenslve Program Proposals } \\
\hline \multicolumn{8}{|l|}{ MRPR Proposals } \\
\hline AWEA ot. al. & statewide & 2 phases $\mathrm{OK}$ & st. or privale agency & incl. in energy price & no sunset & ann. w/ 3-mo, true-up & no provision \\
\hline IEP & regulated providers & only need CPUC & UDCs & line item charge & no sunset & ann. w/ 3-mo, true-up & no provision \\
\hline NCPA & statewide & only legislative & CEC & incl. in energy price & no sunset & annual & no provision \\
\hline SCE/PG\&E & statewide & 2 phases, need both & state agency & incl. in energy price & revisit 2000 & ann. w/ 3-mo. true-up & no provision \\
\hline SMUD & statewide & only legislalive & pow. ex./ISO & incl. in energy price & no sunset & not specified & no provision \\
\hline \multicolumn{8}{|l|}{ Surcharge Proposal } \\
\hline EDF et. al. & statewide & 2 phases OK & slate agency & surcharge & 5 years & continuous & no provision \\
\hline \multicolumn{8}{|l|}{ Adjunct Proposals } \\
\hline $\begin{array}{l}\text { Biogas Working Group } \\
\text { CalSEIA et. al. }\end{array}$ & $\begin{array}{l}\text { not addressed } \\
\text { statewide }\end{array}$ & $\begin{array}{l}\text { not addressed } \\
\text { not addressed }\end{array}$ & $\begin{array}{l}\text { state agency } \\
\text { state agency }\end{array}$ & $\begin{array}{l}\text { incl. in energy price } \\
\text { several oplions }\end{array}$ & $\begin{array}{l}\text { no sunset } \\
\text { no sunsel }\end{array}$ & $\begin{array}{l}\text { not addressed } \\
\text { not addressed }\end{array}$ & $\begin{array}{l}\text { no provision } \\
\text { specified band }\end{array}$ \\
\hline
\end{tabular}

4. Renewable Credils and Markets

\begin{tabular}{|c|c|c|c|c|c|c|}
\hline Proposal & $\begin{array}{l}\text { Renewable } \\
\text { Credits }\end{array}$ & $\begin{array}{l}\text { Contract Terms } \\
\text { for Credits }\end{array}$ & $\begin{array}{l}\text { Credlits from QFs } \\
\text { with ExIsting PPAs }\end{array}$ & $\begin{array}{l}\text { Cost } \\
\text { Cap }\end{array}$ & $\begin{array}{l}\text { Compliance } \\
\text { Penalties }\end{array}$ & $\begin{array}{c}\text { Use of Funds } \\
\text { Collected }\end{array}$ \\
\hline \multicolumn{7}{|c|}{ Comprehensive Program Proposals } \\
\hline \multicolumn{7}{|l|}{ MAPR Proposals } \\
\hline $\begin{array}{l}\text { AWEA ol. al. } \\
\text { IEP }\end{array}$ & $\begin{array}{l}\text { RECs + BECs } \\
\text { RECs }\end{array}$ & $\begin{array}{l}\text { spot to long-term } \\
\text { spot to long-term }\end{array}$ & $\begin{array}{l}\text { generator except during lixed price period } \\
\text { generator except during fixed price period }\end{array}$ & $\begin{array}{c}\$ 0.0275 / k W h \text { REC cap } \\
\text { No Cap }\end{array}$ & $\begin{array}{l}\$ 0.06 / k \text { Wh REC shorlfall } \\
\text { via PBP }\end{array}$ & $\begin{array}{c}\text { purchase RECs } \\
\text { NA }\end{array}$ \\
\hline NCPA & RCCs & spot & negotiale between parties & No Cap & 1 millrelail kWh & renewable R\&D \\
\hline SCE/PG\&E & RECs & spot, bilateral & ulility during entire contract term & $\$ 0.002 / \mathrm{kWh}$ REC cap & administrative & CTC or now renew. \\
\hline \multicolumn{7}{|l|}{ Surcharge Proposal } \\
\hline \multicolumn{7}{|l|}{ Adjunct Proposals } \\
\hline $\begin{array}{l}\text { Biogas Working Group } \\
\text { CalSEIA et. al. }\end{array}$ & $\begin{array}{l}\text { GECs } \\
\text { ETCs }\end{array}$ & $\begin{array}{l}\text { not specilied } \\
\text { spot to long-term }\end{array}$ & $\begin{array}{l}\text { not addressed } \\
\text { not addressed }\end{array}$ & $\begin{array}{l}\text { GEC: } 2 \times \text { AEC price } \\
\text { sel cap on ETC }\end{array}$ & $\begin{array}{l}\text { not addressed } \\
\text { not addressed }\end{array}$ & $\begin{array}{l}\text { not addressed } \\
\text { not addressed }\end{array}$ \\
\hline
\end{tabular}


Table 2: Features of Proposals to Implement The CPUC Renewables Policy

1. Program Obligation

\begin{tabular}{|c|c|c|c|c|c|c|c|}
\hline Proposal & $\begin{array}{l}\text { Program } \\
\text { Based on }\end{array}$ & $\begin{array}{l}\text { Basis for } \\
\text { Initial MRPR }\end{array}$ & $\begin{array}{l}\text { Inllial MRPR with Full } \\
\text { Implementation }\end{array}$ & $\begin{array}{c}\text { Increase In MRPR } \\
1998-2000 \\
\end{array}$ & $\begin{array}{l}\text { MRPR with CPUC } \\
\text { Implementation }\end{array}$ & $\begin{array}{c}\text { MRPR } \\
\text { Applled to }\end{array}$ & $\begin{array}{c}\text { Specified } \\
\text { Technology Bands }\end{array}$ \\
\hline \multicolumn{8}{|c|}{ Comprehensive Program Proposels } \\
\hline \multicolumn{8}{|l|}{ MRPR Proposals } \\
\hline AWEA et. al. & energy & $90 \%$ of 1993 level & $11.6 \%$ & $+0.2 \% / y$ & adjust upward & energy sales to users & Biomass \\
\hline IEP & energy & 1993 level + BRPU & not available & None & CPUC only needed & energy sales to users & Biomass \\
\hline NCPA & capacily & ER '94 proj. for 1998 & $18.0 \%$ & None & state-wide only & ave. monthly peak cap. & None \\
\hline SCERPG\&E & energy & approx. level of 1990s & $10.0 \%$ & None & same as state-wide & energy sales to users & None \\
\hline SMUD & energy & 1994 level & $21.0 \%$ & None & slate-wide only & energy sales to users & None \\
\hline \multicolumn{8}{|l|}{ Surcharge Proposal } \\
\hline EDF et. al. & energy & NA & NA & NA & NA & NA & None \\
\hline \multicolumn{8}{|l|}{ Adjunct Proposals } \\
\hline $\begin{array}{l}\text { Biogas Working Group } \\
\text { CalSEIA et. al. }\end{array}$ & $\begin{array}{l}\text { energy } \\
\text { energy }\end{array}$ & $\begin{array}{l}\text { not addressed } \\
\text { not addressed }\end{array}$ & $\begin{array}{l}\text { not addressed } \\
\text { not addressed }\end{array}$ & $\begin{array}{l}\text { not addressed } \\
100 \mathrm{GWh} / \mathrm{y}\end{array}$ & $\begin{array}{l}\text { not addressed } \\
\text { not addressed }\end{array}$ & $\begin{array}{l}\text { not addressed } \\
\text { not addressed }\end{array}$ & $\begin{array}{c}\text { Biogas } \\
\text { Emerging Technolagies }\end{array}$ \\
\hline
\end{tabular}

$\quad$ 2. Program Ellglbility

\begin{tabular}{|c|c|c|c|c|c|c|c|}
\hline Proposal & $\begin{array}{l}\text { Hydro } \\
\text { Ellgibility }\end{array}$ & $\begin{array}{l}\text { Ellgibillty of Non- } \\
\text { Calli. Renewables }\end{array}$ & $\begin{array}{l}\text { Ellglbility of Bulk } \\
\text { Utility Renewables }\end{array}$ & $\begin{array}{l}\text { Ellgibillty of UDC } \\
\text { Dist. Renewables }\end{array}$ & $\begin{array}{l}\text { Ellglbility of } \\
\text { Existing afs }\end{array}$ & $\begin{array}{l}\text { Ellgiblity of Power } \\
\text { Gen. for On-Site Use }\end{array}$ & $\begin{array}{l}\text { Ellgibility } \\
\text { of Hybrids }\end{array}$ \\
\hline \multicolumn{8}{|c|}{ Comprehensive Program Proposals } \\
\hline \multicolumn{8}{|l|}{ MRPR Proposals } \\
\hline AWEA et. al. & not eligible & BECs Calit. only & eligible & needs resolution & eligible & not eligible & RECs if qualify \\
\hline NCPA & ellgible & not eligible & eligible & eligible & eligible & not eligible & RCCs if qualify \\
\hline SCE/PGRE & not eligible & ellgible & eligible & needs resolution & eligible & not eligible & pro-rate RECs \\
\hline SMUD & eligible & ellgible & ellgible & not addressed & eligible & eligible & pro-rate RECs \\
\hline \multicolumn{8}{|l|}{ Surcharge Proposal } \\
\hline EDF et. al. & not eligible & eligible & after CTC retired & needs resolution & post $12 / 20 / 95$ only & not eligible & pro-rate credits \\
\hline \multicolumn{8}{|l|}{ Adjunct Proposals } \\
\hline Blogas Working Group & not eligible & $\begin{array}{l}\text { eligible } \\
\text { ellipible }\end{array}$ & $\begin{array}{l}\text { eligible } \\
\text { elligible }\end{array}$ & not addressed & $\begin{array}{c}\text { eligible } \\
\text { not elliajble }\end{array}$ & not eligible & pro-rate RECs \\
\hline
\end{tabular}


Table 2: Features of Proposals to Implement The CPUC Renewables Policy (cont.)

3. Program Administration

\begin{tabular}{|c|c|c|c|c|c|c|c|}
\hline Proposal & $\begin{array}{l}\text { Application } \\
\text { of Program }\end{array}$ & $\begin{array}{l}\text { Implementation in } \\
\text { Phases Permitted }\end{array}$ & $\begin{array}{c}\text { Administrator } \\
\text { (full implementation) }\end{array}$ & $\begin{array}{l}\text { - Funding } \\
\text { Mechanism }\end{array}$ & $\begin{array}{l}\text { Program } \\
\text { Lifetime } \\
\end{array}$ & $\begin{array}{l}\text { Perlod to Determine } \\
\text { Compllance }\end{array}$ & $\begin{array}{c}\text { Commerciallzation } \\
\text { Support }\end{array}$ \\
\hline \multicolumn{8}{|c|}{ Comprohensive Program Proposa/s } \\
\hline \multicolumn{8}{|l|}{ MRPR Proposais } \\
\hline AWEA et. at. & State-Wide & 2 phases OK & st. or private agency & $\begin{array}{l}\text { Incl. in energy price } \\
\text { line item charge }\end{array}$ & $\begin{array}{l}\text { No Sunset } \\
\text { No Sunset }\end{array}$ & $\begin{array}{l}\text { ann. w/3.mo, true-up } \\
\text { ann.w/3.mo, true-up }\end{array}$ & $\begin{array}{l}\text { no provision } \\
\text { no provision }\end{array}$ \\
\hline IEP & Regulated Providers & only need CPUC & $\begin{array}{l}\text { UDCS } \\
\text { CEC }\end{array}$ & incl. in energy price & No Sunset & annual & no provision \\
\hline SCE/PG\&E & State-Wide & 2 phases, need both & state agency & ind. in energy price & Revisit 2000 & ann. w/ 3.mo. true-up & no provision \\
\hline SMUD & State-Wide & only legislative & pow. ex./ISO & incl. in energy price & No Sunset & not specified & no provision \\
\hline $\begin{array}{l}\text { Surcharge Proposal } \\
\text { EOF et. al. }\end{array}$ & State-Wide & 2 phases $\mathrm{OK}$ & state agency & surcharge & 5 years & continuous & no provision \\
\hline \multicolumn{8}{|l|}{ Adjunct Proposals } \\
\hline $\begin{array}{l}\text { Biogas Working Group } \\
\text { CaISEIA et. al. }\end{array}$ & $\begin{array}{l}\text { not addressed } \\
\text { State-Wide }\end{array}$ & $\begin{array}{l}\text { not addressed } \\
\text { not addressed }\end{array}$ & $\begin{array}{l}\text { state agency } \\
\text { state agency }\end{array}$ & $\begin{array}{l}\text { incl. In energy price } \\
\text { several options }\end{array}$ & $\begin{array}{l}\text { No Sunset } \\
\text { No Sunset }\end{array}$ & $\begin{array}{l}\text { not addressed } \\
\text { not addressed }\end{array}$ & $\begin{array}{l}\text { no provision } \\
\text { specified band }\end{array}$ \\
\hline
\end{tabular}

4. Renewable Credits and Markets

\begin{tabular}{|c|c|c|c|c|c|c|}
\hline Proposal & $\begin{array}{c}\text { Renewable } \\
\text { Credits }\end{array}$ & $\begin{array}{l}\text { Contract Terms } \\
\text { for Credits }\end{array}$ & $\begin{array}{c}\text { Credits from QFs } \\
\text { with Existing PPAs }\end{array}$ & $\begin{array}{l}\text { Cost } \\
\text { Cap }\end{array}$ & $\begin{array}{c}\text { Compllance } \\
\text { Penalties }\end{array}$ & $\begin{array}{l}\text { Use of Funds } \\
\text { Collected }\end{array}$ \\
\hline \multicolumn{7}{|c|}{ Comprehensive Program Proposals } \\
\hline \multicolumn{7}{|l|}{ MRPRProposals } \\
\hline AWEA et. al. & RECs + BECs & spot to long-term & generator except during fixed price period & 2.75 \&lWh REC cap & 6 flkWh REC shortfall & purchase RECS \\
\hline IEP & RECS & spot to long-term & generator except during fixed price period & No Cap & vla PBR & NA \\
\hline NCPA & RCCs & spot & negotiate between parties & No Cap & 1 mill/retail $\mathrm{kWh}$ & renewable R\&D \\
\hline SCE/PG\&E & RECS & spot, bilateral & utility during entire contract term & $2 \phi / \mathrm{hWh}$ REC cap & administrative & CTC or new renew. \\
\hline SMUD & RECS & not specified & utility during entire contract term & not specified & not addressed & renewables develop. \\
\hline \multicolumn{7}{|l|}{ Surcharge Proposal } \\
\hline EDF et. al. & production credits & $10 \cdot y r s$ & not eligible for credits & set for program & NA & production credits \\
\hline \multicolumn{7}{|l|}{ Adjunct Proposals } \\
\hline $\begin{array}{l}\text { Biogas Working Group } \\
\text { CalSEIA et. al. }\end{array}$ & $\begin{array}{l}\text { GECs } \\
\text { ETCs }\end{array}$ & $\begin{array}{c}\text { not specified } \\
\text { spot to long-term }\end{array}$ & $\begin{array}{l}\text { not addressed } \\
\text { not addressed }\end{array}$ & $\begin{array}{l}\text { GEC: } 2 \times \text { REC price } \\
\text { set cap on ETC }\end{array}$ & $\begin{array}{l}\text { not addressed } \\
\text { not addressed }\end{array}$ & $\begin{array}{l}\text { not addressed } \\
\text { not addressed }\end{array}$ \\
\hline
\end{tabular}




\section{Legend for Table: Features of Proposals to Implement the CPUC Renewables Policy}

\section{Program Obligation}

Program Based on: A minimum renewables purchase requirement (MRPR) standard, or surcharge-funded production credits, can be denominated in either energy units $(\mathrm{kWh})$ or capacity units $(\mathrm{kW})$.

Basis for Initial MRPR: Most of the proposals have, as one of their objectives, the preservation (in some form) of the existing renewable energy industry in the state. This category shows the basis or objective for the setting of the proposed initial MRPRs by the various proposers, for proposals including an MRPR.

MRPR with Full Implementation: The proposed MRPR standard (for those proposals containing an MRPR standard), which in all cases is a percentage of a defined block of electricity that must be renewable. With full implementation means that, for those proposals that permit a two-phase implementation (initially by the CPUC, subsequently by the legislature), this column indicates the level of the proposed standard with the program fully enacted. Most of the proposals would adjust their proposed standard to meet their stated program goals, subject to a better understanding of current levels of renewables production in Califomia. The SCE/PG\&E proposal proposes a specific percentage for the MRPR.

MRPR with CPUC Implementation: For those proposals that provide for a two-phase implementation, beginning with enactment by the CPUC for the regulated electricity market, and following with state-wide implementation, this column indicates the level of the standard proposed for enactment during the first (CPUC only) phase of the program.

MRPR Applied to: The category(ies) of electric energy or capacity that the MRPR standard is applied to, for each entity that is required to meet the obligation.

Increase in MRPR 1998 - 2000: The MRPR proposed for the first year of the program may or may not be adjusted during the first several years of the program. In all cases, load growth leads to an increase in the quantity of renewable energy required.

Specified Technology Bands: Some of the proposals include special-qualification sub-bands within the MRPR in order to guarantee minimum levels of specific technologies or classes of technologies. Band requirements, like the overall MRPR, must be individually complied with.

\section{Program Eligibility}

Hydro Eligibility: All proposals define biomass, geothermal, solar electric (thermal and photovoltaic), and wind technologies as qualifying renewables, in accordance with the CPUC restructuring decision. Some proposals include hydroelectric generation in the mix of eligible generating options, others do not.

Eligibility of Non-Calif. Renewables: Proposals may or may not restrict renewable energy generated from out-of-state sources from participation in the program.

Eligibility of Bulk Utility Renewables: Utility-owned renewables, mainly geothermal and, if included, hydro, may or may not be eligible for participation in the program.

Eligibility of Existing QFs: Existing QF renewables may or may not be eligible for participation in the program.

Eligibility of UDC Dist. Renewables: Distributed renewables owned by a UDC or its affiliates may or may not be eligible for participation in the program. Distributed generation is small-scale power installed in the distribution system or on a customer's site. It can capture T\&D benefits and/or serve local loads.

Eligibility of Power Gen. for On-Site Use: Renewable energy that is used by the producer for on-site applications may or may not be eligible to receive RECs or production credits. Surplus or net power sold by self-generators to others is eligible to receive credits in all of the proposed programs. The entries in this column pertain to the eligibility of renewable power that is used on-site, and not sold to a distributor or user.

Eligibility of Hybrids: Hybrid generators, which use both renewable and non-renewable energy sources, require special rules to determine qualification for RECs or production credits. Some proposals give full renewable credit to hybrids that derive more than $75 \%$ of their energy from renewables, while others give only pro-rated credit. For hybrids that derive less than $75 \%$ of their energy from renewables, some proposals give pro-rated credit, others give no credit. 


\section{Program Administration}

Application of Program: Renewables support programs can be designed for state-wide application, or for application only to electric services providers subject to CPUC regulation.

Implementation in Phases Permitted: A renewables support program can be imposed by the CPUC only on electric services providers under its jurisdiction. Application of the program to unregulated utilities will require legislative action. Some proposals allow for a two-phase impiementation of the renewables program, first by the CPUC, then by the legislature. Some of the proposals are designed for state-wide implementation only. One proposal is designed for full implementation by the CPUC, without the need for legislative action. Of the proposals that permit a two-phase implementation, one proposal calls for program cancellation if state-wide implemention is not achieved, others would maintain the program at the CPUC implementation level if legislation is not forthcoming.

Administrator (full implementation): Various state or private agencies are proposed to administer the programs. The proposed administrator listed in the table assumes full implementation for those proposals that provide for a twophase implementation.

Funding Mechanism: A renewables support program is expected to be more costly than a restructured market lacking a renewables program. The cost of the renewables program can be rolled into the price of electricity, or it can be assessed as a line-item or surcharge to electricity customers.

Program Lifetime: The renewables program may be imposed permanently (with no sunset date), imposed for a fixed term, or imposed for a limited term subject to program review and reauthorization.

Period to Determine Compliance: Compliance with program requirements can be determined over various periods of time, with a true-up period allowed or not allowed.

Commercialization Support: Proposals may or may not include provisions for assisting emerging renewable energy technologies that have moved beyond the RD\&D stage, but whose cost is not yet competitive with commercially mature renewables.

\section{Renewable Credits and Markets}

Renerrable Credits: The various proposals contain a variety of different types of renewable energy credits, including: BECs (biomass energy credits), GECs (greenhouse environmental credits), ETCs (emerging technology credits), production credits, RCCs (renewable capacity credits), and RECs (renewable energy credits).

Contract Terms for Credits: Sales of renewables credits can be for contract terms ranging from spot market to long-term commitments. Some of the proposals specify the contract terms they foresee for credits, other proposals do not address this issue.

Credits from QFs with Existing PPAs: Many existing renewable $Q F s$ have long-term power purchase agreements (PPAs) with utilities. Assignment of credits associated with renewable energy sold under existing long-term PPAs may be to the generator or to the purchaser. Some of the proposals propose to assign credits to the buyer for the entire terms of the contracts. Others propose to assign the credits to the buyer when the energy is sold under the fixed-price (FP) schedules in the old ISO4 contracts, and to the generator when the energy is sold under SRAC rates.

Cost Cap: The cost of compliance with the renewables program requirements may or may not be capped. This category shows the proposed cap on the annual cost of program compliance, if the cost is capped. For the adjunct proposals, the category shows the cap on the cost of the adjunct program only.

Compliance Penalties: Penalties for non-compliance with the renewables program may or may not be included in individual proposals.

Use of Funds Collected: Proposals may or may not specify how to use penalty or compliance funds collected, in cases where the proposals include provisions for the collection such funds. 
All the MRPR proposals place compliance obligations on electric services providers. The IEP proposal imposes the obligations on the UDCs only; the others impose the obligations on all providers. Two approaches for determining compliance obligations during each defined compliance period are proposed. Several proposals require obligated parties to acquire a specified quantity of RECs during each compliance period that is a percentage of their sales for that period. Because exact sales quantity during a compliance period cannot be determined in advance, these proposals provide for a true-up period after each compliance period. The NCPA proposal provides for compliance obligations to be determined retrospectively, based on the obligated entities' average sales volumes during the previous 12 months. This approach facilitates the parties' REC planning, as they know at the beginning of each compliance period what their REC obligation will be for that period.

Each proposal uses one or both of two primary tools to adjust the amount of renewable energy production associated with the proposed program:

I. A standard (the MRPR), which specifies the minimum amount of renewable energy that must be produced.

2. A program cost allocation or cost cap that determines the (maximum) amount that will be spent to support renewable energy production within the program.

The CPUC's decision on restructuring recommends the use of an MRPR standard to achieve its objectives for renewable energy. The decision leaves open the issue of whether to impose a cost cap on the program. The IEP proposal relies entirely on the use of an MRPR standard for meeting the commission's objectives; the EDF et al. proposal relies entirely on the use of an administratively determined program cost allocation. Proposals that employ both an MRPR standard and a cost cap become blends of the two approaches, with renewable energy production that can be manipulated by adjusting either variable. If the cost cap is set lower than the marginal price of RECs needed to fulfill a mandated MRPR, the MRPR program standard will probably not be achieved. The challenge for the commission and the legislature is to balance program cost and the level of renewable energy production desired.

All comprehensive renewables policy implementation proposals except for the NCPA proposal are based on creating obligations for the purchase of renewable energy, as measured in kilowatt-hours of electricity delivered to California users. In any given period of time, the MRPR percentage of defined energy must be generated from renewable generating sources, or in the case of the EDF et al. proposal, renewable energy production credits are distributed to renewable energy generators based on their energy production. Denominating a program with energy units ensures that the amount of electricity produced from renewable sources, rather than the amount of renewable generating capacity in service, is the objective of the program. This is based on proposers' conceptions that renewables make their greatest contribution by their operation, not just by their online availability. Also, monitoring a program based on energy units is straightforward, as electric energy is routinely metered for sales and transfers through the grid.

The NCPA proposal is based on creating an obligation for an MRPR percentage of generating capacity from renewable sources, as measured in kilowatts. The proposal includes a requirement that suppliers of qualifying renewable capacity maintain a minimum level of energy generation commensurate with the generating technology in question. The NCPA proposal has the advantage that the obligation for any given period is based on the average monthly capacity used for the previous year, and thus is determinable before each compliance period begins. Entities that are obligated to amass capacity credits know beforehand how many credits they must acquire, and no true-up period is required. The capacity-credit approach is designed to minimize the uncertainty associated with annual variations in the availability of intermittent renewable generating sources (solar, wind, and especially hydrogen). Intermittent generators are required to bid their capacity at a level that allows qualification with regard to required energy production in poor resource years, or face de-rating if they fail to perform. 
The requirement in the NCPA proposal that a renewable generating source provide a minimum annual amount of energy to qualify as having provided its certified capacity to the system minimizes the difference between an energy-based MRPR and the proposed capacity-based MRPR. For example, if the administering agency determines that a given renewable technology must operate at a load factor of $80 \%$ to qualify as having met its capacity provision obligation, bidding a generating unit using this technology at the level of $10 \mathrm{MW}$ of capacity credits is equivalent to bidding a commitment of $70,000 \mathrm{MWh}$ of energy to be produced during 1 year $(10 \mathrm{MW} \times 8760$ $\mathrm{h} / \mathrm{yr} \times \mathrm{0.8}$ ). A capacity credit program that lacks this minimum production requirement would not ensure the same level of renewable energy production as the NCPA proposal or the energy-based proposals.

Some participants argue that a weakness of the capacity credit approach tied to a minimum production level set differently for each renewable energy technology is that the resulting values of the credits, on a per kilowatt-hour basis, would vary greatly. For example, if biomass generators were required to produce at $80 \%$ capacity, and wind generators at $25 \%$, if a capacity credit were valued at $\$ 100 / \mathrm{MW}$ by the market, the biomass generator would receive a capacity value of $\$ 0.014$ for each kilowatt-hour produced subject to capacity credit qualification, and the wind generator would receive a value of $\$ 0.045 / \mathrm{kWh}$. In other words, they argue that compared to a sys- tem based on energy credits, the capacity credit approach proposed by NCPA favors renewable generating technologies that operate at inherently lower capacity factors, and thus would secure for the market fewer kilowatthours of renewable energy per dollar cost of the program.

NCPA believes that the relevant issue for the state's renewable program is not the arithmetic of renewable credits, but the income stream represented by the combination of energy sales and credit sales. The high capacity-factor renewables have more energy to sell, and thus earn more annual revenue from such sales. They also have a greater annual output of energy over which to amortize their capital costs. They will receive a lower per-kilowatthour value for their capacity credits, but the significant issue is whether the total income stream is sufficient to induce continued operation of facilities and prudent new investment. The capacity credit approach helps put low capacity-factor technologies in a position to compete in the market.

MRPRs and Program Goals. All proposals based on the MRPR approach set the initial level of the statewide standard at a level that is based to some degree on the average statewide level of renewable energy generation at the time when the initial electric utility restructuring decision was made by the CPUC (April 1994). Two proposals, IEP and NCPA, would set the initial MRPR at a level intended to obligate the amount of renewables that would have been achieved when the overall restructuring program was expected to be enacted (1998), based on production that probably would have occurred had the biennial resource planning process been completed as originally envisioned. The SMUD proposal sets the initial level at the level of renewable energy produced in 1994 , and the renewables industries proposal sets the level at $90 \%$ of the level in 1993 , with the $10 \%$ reduction adopted in an effort to ensure competition among renewables. The SCE/PG\&E proposal attempts to achieve approximately the level of renewables production the state experienced during the first half of the 1990s. Most MRPR proposals estimate the MRPR level that would achieve their program objectives, but state that the actual standard adopted should be based on achieving the intended goal, rather than on the actual number offered in the proposal. The exception is the SCE/PG\&E proposal, which proposes to adopt $10 \%$ as the numerical standard. To adjust the initial MRPRs to meet stated program objectives, a reliable data set of renewable energy use in California during the early 1990 s would have to be established. The Renewables Working Group was unable to produce a verifiable database that all participants could endorse. This is an appropriate area for future commission inquiry.

All the proposals attempt to maintain statewide levels of renewables production at levels consistent with those of the early 1990s, but applying the proposed MRPRs uniformly to all providers, or to all regulated providers, imposes very different implications for individual providers. San Diego Gas and Electric, for example, would have to increase its renewables purchases, either directly or by acquiring tradable RECs, at least tenfold to comply with the proposed MRPR standards. Only the IEP proposal provides for a transition strategy, in which initial 
MRPRs for each UDC (the sole obligates in this program), are set consistent with current levels of renewables in their individual service territories.

Most proposals anticipate maintaining the initial MRPR at a constant level for the first 3 years, pending an expected review of the renewables program at that time. In this case the total requirement for renewables would change in proportion to changes in total energy consumption during the period (or more exactly, changes in those categories of energy consumption to which the MRPR is applied), but the renewable percentage would remain fixed. The exception to this is the renewables industries proposal, which includes a provision to increase the MRPR by $0.2 \%$ per year during the first 3 years of the program. The renewables industry proposal is the only one that purposely sets the initial MRPR level below the amount of renewables produced in 1993 to ensure competition, so even after 3 years of an increasing MRPR (at $0.2 \% / y r$ ), the statewide level of the renewables program obligation will remain below the pre-restructuring level.

Generation Technologies Included in the Programs. California Public Utilities Code Section 701.1(a) lists as renewable generation technologies biomass (solid fuel and biogas), geothermal, solar (thermal electric and PV), and wind. Although unquestionably renewable, hydroelectric generation is not explicitly included. The inclusion of new or current hydrogen generation in a renewables support program is a matter of contention among the parties to the Renewables Working Group. Two comprehensive program proposals, NCPA and SMUD, include hydrogen among the eligible technologies; the other four exclude it.

Some participants have suggested that including hydroelectric generation in a renewables support program presents philosophical and practical issues. The advocates of the including hydrogen observe that these issues are not unique to hydroelectric generation. The major philosophical issue is the commercial and competitive status of hydroelectric generating technology. Hydrogen technology is fully mature and competitive with other forms of electricity generation. One question is whether hydrogen should be given the same incentives extended to the other renewables. This factor is recognized by the SMUD proposal, which includes hydrogen as a renewable generating option for meeting the MRPR obligation, but prohibits the trading of credits associated with extant hydrogen generators (those commissioned before December 20, 1995). All other renewable energy credits are tradable in the SMUD program. Hydrogen proponents observe that biomass and geothermal technologies are also technically mature. Furthermore, operational constraints placed on hydrogen facilities to enhance environmental values affect their competitiveness in ways that parallel the uncertainties associated with fuel availability and price volatility for biomass and geothermal energy systems.

Some practical problems associated with including hydroelectric generation in a renewables support program are:

- Many hydrogen generators are multipurpose facilities that provide water supply, flood control, and recreational amenities in addition to power generation. Including systems of this kind in the renewables program risks subsidizing these nonenergy functions. Similar considerations apply to biomass facilities, which provide ancillary waste disposal services.

- If out-of-state hydrogen generators are deemed eligible, Northwest hydrogen sources might squeeze nonhydrogen renewables out of the market. To address this concern the NCPA proposal excludes out-ofstate generating facilities from participating in the program, and the SMUD proposal prohibits the trading of credits associated with extant hydrogen facilities.

- Year-to-year fluctuations in hydrogen availability, which tend to be more extreme than fluctuations in other renewable energy sources, will make the timely acquisition of RECs more difficult for entities required to meet MRPR-based standards if the standard is based on energy production rather than operational capacity. 
Competition and Diversity of Renewable Generating Sources. Renewable energy generating resources are a disparate collection of technologies, each of which has its own combination of characteristics and needs to contribute to the state's electric system. For example, some renewables, such as solar electric and wind, are dominated by high capital cost, no fuel cost, and low operating cost; others, such as biomass and geothermal, have a more conventional combination of capital and operating costs. Some renewables can be operated in a full or partial load-following mode; others, notably solar electric and wind, provide intermittent power whose output profile is uncontrollable and not synchronizable to consumer demand. In addition, although all renewables may provide environmental, economic, and diversity benefits to California, the costs and benefits associated with each technology vary considerably.

There is an open question among members of the working group as to whether various renewables can compete successfully with each other, or whether head-to-head competition would eliminate some of the current or emerging renewable generating sources from the system. There is also disagreement as to whether, from a public policy perspective, competition among the renewables should be encouraged or discouraged. The CPUC restruc- turing decision discusses the appropriateness of imposing individual technology bands to ensure its diversity goals for renewables.

Two comprehensive program proposals, renewables industries and IEP, include a provision for a special band to support one specific renewable technology: solid-fuel biomass. In these proposals, entities that are obligated to acquire a given quantity of RECs will be further obligated to ensure that a defined minimum fraction of the total REC obligation is contributed by biomass generating sources. The rationale is that biomass technologies provide an especially valuable package of environmental benefits (including waste disposal services) that are unique among the renewables, and biomass has difficulty competing with other renewables that inherently have much lower operating costs. Thus the renewables industries and IEP proposals consider preserving a minimum level of biomass power generation by creating a specified technology band for biomass a reasonable additional program cost.

Both adjunct proposals, BWG and CaISEIA et al., propose an additional mechanism to support selected technologies. BWG proposes a mechanism that would be geared to the mitigation of one specific environmental insult the emission of greenhouse gases associated with treating and disposing of solid wastes. BWG's rationale is that biogas power generation provides a unique environmental service (the additional mitigation of greenhouse gas emissions by reducing methane emissions), and, in the proposers' view, asking electricity customers to pay extra to receive this particular environmental service is reasonable.

BWG does not use the conventional band mechanism to promote biogas production because, it argues, banding is most effective in preserving a level of production already achieved, and in the case of developing biogas generating resources, there is a potential to increase the installed capacity several fold. Instead, the proposal creates a new category of credits called "greenhouse environmental credits" (GECs). Each kilowatt-hour of electricity produced from biogas produces one associated REC and one associated GEC. Each GEC has a value equal to that of an REC, and provides a significant additional incentive to produce electricity from biogas. To avoid outcompeting other renewable energy sources with the increased credit allocation to biogas generators, BWG proposes that increases in the installed capacity of biogas generators should be accompanied by a commensurate increase in the MRPR. The intent is to leave the requirement for non-biogas renewables unaffected by the level of biogas-generated power.

CalSEIA et al. propose a special band or surcharge that would be used to promote the commercialization of emerging renewable generating technologies that have moved beyond $R \& D$, but are not yet competitive with the lowest-cost renewables in the market. A variety of solar technologies, such as PV and dish-Stirling engines, and other renewable technologies, fit this category. CalSEIA et al. propose temporary support of such technologies at a higher level than the expected value of the credits associated with "conventional" renewables to help them 
move down the technology commercialization curve and become competitive with conventional renewables and other generating sources. The special band or surcharge could be added onto any of the comprehensive program proposals included in this report.

The six comprehensive program proposals do not include provisions for commercializing emerging technologies, but argue that the CPUC's renewables policy is intended to be a support program for competitive renewables sources, and not a mechanism to support technology commercialization. On the other hand, there is currently no other mechanism to provide the type of commercialization support that is the objective of the CalSEIA et al. adjunct proposal. Because the commercialization band will probably not engender the level of competition expected within the MRPRs of the full program proposals, commercialization might alternatively be pursued via a surcharge-funded program that runs as an adjunct to whichever renewables program is adopted. One option proposed by CalSEIA et al., a commercialization surcharge program, would be compatible with any of the comprehensive program proposals, whether the basic program is based on an MRPR or on surcharge-funded production credits. If it is added on to a surcharge-funded program, determining the proportion of the total funds collected that would be allocated to emerging technologies becomes an administrative decision. For rooftop PV, CalSEIA et al. has also proposed that surcharge funds could be administered as part of either the R\&D or energyefficiency program.

\section{Program Eligibility Issues}

Out-of-State Renewables. Most of the comprehensive program proposals do not restrict the participation of outof-state renewable generating sources. Most of the proposers believe that, although restricting the program to in-state renewable generating sources would be economically desirable for California, any such restrictions would be contrary to the Commerce Clause of the U.S. Constitution, which prohibits restrictions on interstate trade. The exceptions are the renewables industries and NCPA proposals. The NCPA takes the position that restricting participation in the program to in-state renewable generating sources would be both legal and desir- able because renewable generating facilities provide unique local environmental and public health benefits that justify restricting program eligibility to local generating facilities.

The renewables industries proposal adopts a narrower version of this rationale. It places no restrictions on out-ofstate generators in the general RECs market, but restricts participation in the biomass credits market to in-state biomass generators. The proposal recognizes Commerce Clause considerations, but believes that, in the case of the biomass set-aside, there may be a enough in-state interest to allow the restriction to be applied. The rationale is that establishing this special band in the first place is to secure for the state the waste disposal bene- fits of biomass power generation, such as reductions in open agricultural buming, landfilling requirements, and forest fire risks via the removal of excess fuel from the forest. These benefits accrue to California if biomass facilities use only biomass that originates in California. The Renewables Working Group is unable to provide legal guidance to the CPUC on Commerce Clause issues.

UDC-Owned Renewables. One renewable energy application that presents a special set of regulatory issues is UDC-owned distributed generation. Distributed generation takes the form of smaller disbursed generating facilities located at a customer, utility, or other location. Distributed renewables can include PV, wind, and biomass technologies. Distributed renewable generation could be owned by UDC, customers, or third parties, such as green direct-access providers. At a customer's premises, distributed renewables could include self-generation, third-party on-site generation, or utility generation connected on either side of the meter. 
Some utilities and others have proposed that utility-owned distributed generators be considered transmission and distribution (T\&D) plants and therefore exempt from the unbundling of generation from T\&D." This would permit UDCs to use distributed renewables to substitute for T\&D expansion, in effect "leapfrogging" T\&D congestion by moving their generating resources closer to customers. The potential of the UDCs to crosssubsidize their distributed generation with savings on the T\&D side is also an issue in restructuring, as is the locational market power concern related to the UDCs' unique status among potential distributed generators as the owners of the distribution system.

Another potential issue is the power exchange purchase requirement of UDCs. Under restructuring, utilities are required to obtain energy through the power exchange. However, distributed generation may be unsuited to bidding into a power exchange because of transaction costs, nondispatchability, line losses, or the unfeasibility of wheeling power from distribution to transmission.

The renewables industries, CaISEIA et al., and IEP proposals state that UDC-owned distributed renewables should not qualify for RECs until these issues are resolved. The renewables industries and CalSEIA et al. proposals would accelerate the commercialization of distributed renewables through the pass-through of T\&D benefits to customers and third parties, and through the use of energy efficiency and RD\&D monies. The NCPA proposal would also make UDC-owned distributed renewables eligible for RECs. The EDF et al. and SCE/ PG\&E proposals state that UDC-owned distributed renewables may be eligible for subsidy by surcharge-funded production credits or RECs once competitive transition charge (CTC) recovery is completed and the commission has resolved the functional unbundling and other issues in restructuring. The SMUD and biogas proposals do not address the question of distributed renewables owned by UDCs.

Current Renewables. The five MRPR-based proposals make current utility-owned and qualifying facility (QF) renewable power generators eligible to participate competitively in a renewable credits program. The only exception is the SMUD proposal, which includes hydrogen in the program but prohibits the trading of credits associated with hydrogen generating sources. The authors impose this restriction to limit the market power of hydrogen-generating sources within the overall renewables market. The hydrogen generators are counted toward the renewables obligation of the UDC that distributes their power, but their credits are not transferable.

The EDF et al. production credit proposal excludes current future utility-owned renewables from participating in the surcharge program until CTC issues are resolved and CTC amounts fully collected. Nonutility=owned renewable generating sources would be eligible to participate only if their in-service date is after December 20 , 1995 (the date of the CPUC restructuring decision), or if a facility was substantially redeveloped after that date. As such, under the EDF et al. proposal, current QFs would not be eligible to participate in the surcharge-funded production credit program regardless of whether they continued to sell under power purchase contracts. As currently drafted, this program is designed to encourage the development primarily of new renewables projects.

Renewables Generation for On-Site, Own Use. Some renewable energy generated in California is used on site by the generator"* rather than being sold to the utility companies for distribution and sale. Renewable selfgeneration occurs in two major situations: in nongrid-connected applications for which the cost of grid connection would be more expensive than the cost of installing and operating an on-site renewable generating system, and in grid-connected applications for which the generator supplies its own energy requirements from a

\footnotetext{
"SDG\&E, EPRI, and four utilities outside Califomia are funding a study of legal and regulatory issues connected with this issue. All three California IOUs have conducted ratepayer-funded RD\&D into integrating distributed generation into their T\&D systems. The SCE/PG\&E proposal suggests that RECs be awarded to distributed utility-owned renewable power.

- For purposes of this discussion, power that is used within the renewable generating facility, commonly referred to as parasitic power, is not considered to be self-generation.
} 
combination of the renewable generator and the grid, and supplies net or surplus renewable power to the grid. Renewable self-generation can vary in scale from a $200-\mathrm{W}$ solar home system to a 50-MW biomass cogeneration system associated with a pulp and paper mill.

All the comprehensive renewables program proposals would award RECs (or RCCs or production credits) to the quantities of surplus renewable energy generation that grid-connected self-generators provide through a util- ity meter (eventually) to a customer. Two proposals, IEP and SMUD, would also award RECs for renewably generated power used on site by the generator; the other four would prohibit such power from qualifying for RECs. Those four proposers are concerned that awarding credits to self-generation may be impractical because power consumed on site is not officially tracked or sold through a regulated meter. Hence, the kilowatt-hours of self-generation cannot be verified. Some members of the working group believe that including self-generation in the renewables program might encourage electricity users to avoid public purpose charges and the CTC.

Hybrid Generators. Renewable generating technologies that incorporate heat engines in their systems can operate with renewable and nonrenewable energy sources in a hybrid generating mode. Renewables in this category include biomass, geothermal, and solar thermal electric generation. There are technical, efficiency, and economic reasons why generating facilities that use these technologies choose to hybridize routinely with natural gas as an energy source, both spot and continuously. PURPA allows a renewable generating facility to obtain as much as $25 \%$ of its energy input from nonrenewable sources and maintain its qualifying status as renewable.

To qualify for renewable energy credits, several approaches for treating hybrids, all of which are represented in the six comprehensive program proposals, are possible. The two basic approaches are: (1) prorate the renewable portion of the generator's output for purposes of REC qualification; and (2) set a minimum renewable qualification for the generator and give full REC credit for complying facilities. Three proposals (SCE/PG\&E, SMUD, and EDF et al.) would assign prorated credits for hybrids with any combination of renewable and nonrenewable energy. The renewables industries, IEP, NCPA, and CalSEIA proposals establish a $75 \%$ renewable qualification minimum, and award full renewable credits for generators that meet the minimum renewable rule. The IEP and NCPA proposals would establish a 75\% minimum renewable qualification and would assign no RECs to hybrids that do not meet the minimum qualification rule. The renewables industries proposal allows prorated credits for such facilities.

\section{Program Administration Issues}

Program Administration. The decision on electric utility restructuring expressed a preference for statewide implementation of its renewable energy policy, which can be accomplished only through legislative enactment. Because of jurisdictional considerations, CPUC programs apply only to the investor-owned, regulated electric utility sector. Most proposed comprehensive renewables programs are designated for statewide application, although some allow for a two-phased implementation, beginning with the regulated electric utility sector, and extending in the second phase to the entire electric utility industry via legislative enactment. The renewables industries proposal provides for a two-phase implementation approach that would continue the program at the CPUC level regardless of the status of statewide legislative implementation. The SCE/PG\&E and EDF et al. proposals would allow for initial CPUC implementation, but recommend canceling the program if timely legislative enactment is not achieved. The NCPA and SMUD proposals are designed for implementation at the state level only. The IEP proposal, in an effort to facilitate the implementation of the CPUC's renewables policy, is designed around enactment at the CPUC level only. Statewide application of the program would be welcomed by the IEP, but the program is designed to achieve its full program goals with CPUC implementation.

Renewables industries and SCE/PG\&E provide for a two-phase implementation of the renewables program, but they take different approaches to phase in the program. Renewables industries would apply higher standards during initial CPUC enactment to achieve full program objectives in terms of statewide renewables use within 
the limited context of the regulated electricity sector. Upon statewide enactment, the standards would be adjusted to achieve the same renewables production level over the extended participant base. SCE/PG\&E would set the MRPR standard at 10\% during initial enactment by the CPUC, the same level that would be applied statewide when the program is so extended.

The CPUC's electric utility restructuring program is scheduled to be implemented at the beginning of 1998, with a review of the renewables program expected after the third year of operation. Most proposals contain no sunset date, in order to create the long-term commitment necessary to attract investments in new renewables generating capacity. Several proposals point out that the programs will automatically sunset themselves if and when market conditions make renewables fully competitive with nonrenewable electric generating sources. These proposals do not indicate whether they believe subsidies should continue indefinitely should renewables not be able to compete head-to-head with other generating sources. SCE/PG\&E and EDF et al. suggest that during the program review following the year 2000 a specific determination be made regarding the continuation of the renewables program. EDF et al. proposes to award production credits through a series of five annual auctions. Successful bidders will be awarded contracts for production credits with 10-year terms, beginning with the in-service date of the auction winners.

The comprehensive proposals present several alternatives for administering a renewables program. Four provide for the program to be administered by a state agency (the CEC was named specifically in the NCPA proposal). The renewables industries proposal allows for either a state or private agency to administer. The SMUD proposal calls for the program to be administered by the wholesale power exchange and independent system operator, which will be created as new institutions during the first phase of the implementation of the CPUC's overall restructuring program. The IEP proposal takes a different approach, assigning administrative duties to the UDCs that will be created as part of the restructuring process. The IEP proposal depends on state agencies to provide certification standards and services to the renewables program. The EDF et al. proposal suggests.assigning administrative duties to the California Alternative Energy and Advanced Transportation Financing Authority, but does not preclude the use of other state agencies to provide administrative services for the program.

Compliance and Enforcement. The CPUC restructuring decision calls for the enactment of a renewables program that is supported by effective compliance and enforcement provisions. Each comprehensive proposal takes a different approach to addressing this aspect of the program.

The renewables industries proposal would impose a high, punitive penalty $(\$ 0.06 / \mathrm{kWh})$ on electricity providers that fail to acquire enough RECs to meet their program obligation, with the intention of ensuring full compliance at all times. The penalty is applied to the shortfall in a provider's renewables obligation. Full compliance is further ensured by setting the initial MRPR at a level that can be met with only $90 \%$ of the renewables production actually produced during 1993. The proposal provides cost control by including a cost cap for the RECs $(\$ 0.0275 / \mathrm{kWh})$ and BECs $(\$ 0.0375 / \mathrm{kWh})$. If the program administrator sells credits at the cap price, the funds collected will be used to conduct a secondary auction, purchasing credits from the market at whatever price is offered subject to the availability of funds.

The IEP proposal emphasizes voluntary compliance by non-UDC providers through direct-access green marketing, and requires the UDCs to acquire any additional renewable energy credits necessary to meet the statewide MRPR standard, with their costs billed as a line-item charge to all UDC customers, including direct-access customers. The line-item charge will be applied the same as public purpose charges or the CTC. Direct-access customers of certified green-energy providers will not be assessed the line-item charge. Green-energy certification will require providers to at least meet the MRPR standard in their portfolio of resource supply. The UDCs are responsible for administering the program, and for demonstrating that the MRPR is met. This responsibility will be enforced as one aspect of the PBR regulatory process to which the UDCs will be subject in the restructured electricity market. No penalties are specified, and the program does not have a cost cap. 
The NCPA proposal gives the CEC responsibility to administer and enforce the renewables program. Electricity providers subject to the program are required to surrender the required number of RCCs, or face a penalty payment of $1 \mathrm{mil} / \mathrm{kWh}$ assessed to their entire volume of power sales. The penalty is a cost cap for the program, and all penalty funds collected would be devoted to renewables R\&D. A drawback to a penalty that is assessed to a provider's entire sales volume is that it provides no incentive to achieve partial compliance if a provider cannot achieve full compliance at a cost that is below the cap. In such cases a provider might choose to pay the penalty instead of participating in the program, which could suppress the value of RCCs across the board.

The SCE/PG\&E proposal includes provisions for a $\$ 0.02 / \mathrm{kWh}$ price ceiling to be applied to the shortfall of RECs a provider is obligated to acquire, as well as possible penalties for fraudulent behavior. The ceiling price is intended to be a fee, not a penalty, and to be a cost cap for the renewables program. Funds collected from ceiling payments made in lieu of REC acquisition could be used to reduce the CTC, or to promote the development of new renewables.

The SMUD proposal does not address the issue of penalties and enforcement.

The EDF et al. proposal is based on a surcharge-funded program rather than on the establishment of an MRPR, so enforcement requirements for the program differ from those of the MRPR-based proposals. The program is based on the use of an administratively determined cost to be used to fund renewable technologies. The proposers do not recommend a specific overall funding level, but use as an example a program funding level of $\$ 125$ million, assuming the program is enacted statewide. Compliance incentives or penalties are not expected to be necessary for this type of program. The program funds would be administered by a state agency.

The CaISEIA et al. proposal does not specifically address penalties for noncompliance, but proposes a cost cap on the price of credits for the emerging technologies band. The cap would not be a fixed price, but would be set at some specified multiplier above general REC trading prices. If market price reached the cap, it would trigger the program administrator to sell credits at the cap price and use the proceeds to fund increased renewables generation.

Renewable Credits and Credit Markets. The CPUC's restructuring decision proposes a renewables program based on an MRPR that is intended to be applied statewide to all electricity sales to end users. To facilitate compliance and minimize program cost, the decision envisions the creation of a market for trading renewable energy credits, allowing electricity providers that are deficient in renewable generating resources to fulfill their obligation by purchasing available credits from renewable energy used anywhere in the state. Renewable energy generators benefit by having two commodities to sell, renewable energy and its associated RECs. In addition, the purchasers of renewable energy may benefit from reselling RECs to retail sellers that require additional credits to meet their MRPR requirement. The value of the RECs is intended to provide the above-market increment that renewables generators need to compete in the restructured market. The value of the RECs will be controlled by market competition, assuming a competitive market is engendered by the program. The five MRPR-based proposals offer several alternatives for structuring a competitive REC market.

Most of the proposals are nonspecific about the structure or mechanism of the market that would be created for trading RECs. They would allow a variety of transfer mechanisms to develop, including bilateral contracts, packaged energy and REC sales contracts, long-term contracts, and spot sales. In most proposals, providers of energy to California end users are obligated to acquire a minimum quantity of RECs to satisfy their MRPR obligation, which are to be surrendered to the designated administrator at the end of each compliance period.

The SMUD proposal offers a different approach to operating a REC market, which takes advantage of the creation of the wholesale power exchange and independent system operator (ISO) as part of the restructuring process. The power exchange will purchase all power to be grid distributed as restructuring is implemented, and will 
be responsible for acquiring power at lowest cost. The ISO will be responsible for ensuring that system integrity and reliability standards are maintained. SMUD's proposal suggests that having the exchange also be responsible for acquiring the necessary of RECs, with the cost distributed proportionally to electric service providers as they take power from the exchange for distribution to California end users, would be a natural extension. The exchange would be given the same latitude to balance firm and spot REC purchases as it has for energy purchases. SMUD contends this system would avoid the market power problem that could arise in a market that operates with a limited number of REC purchasers.

RECS from Energy Sold under Current PPAs. All the MRPR proposals agree that the generator of a REC may sell that REC, just as it sells its output of kilowatt-hours. If renewable energy is being sold under long-term PPAs that predate market restructuring, however, the assignment of RECs is far from clear. Because there were no RECs when the PPAs were formulated, there is no specification for REC transfer in these contracts. This is an issue of considerable significance for implementating an MRPR program, as much of the renewable gener- ating capacity that will be available during its enactment will be bound by long-term PPAs, some of which extend more than 20 years beyond the planned restructuring implementation date.

The proposals (renewables industries, IEP, SCE et al., SMUD) that offer a directed solution to the issue of assigning RECs for renewable energy sold under prerestructuring PPAs agree that if renewable energy is being sold under the fixed-price schedules included in standard-offer PPAs (specifically Interim Standard Offer \#4 PPAs with the appropriate selections made), the RECs associated with this energy would be considered to be packaged with the energy, and the property of the purchaser (i.e., the utility).

There is considerable disagreement, however, regarding the assignment of RECs associated with energy being sold under pre-restructuring long-term PPAs, when energy is sold at the SRAC rate, and capacity is sold at longterm levelized contract rates. The renewables industries and IEP proposals assign all RECs associated with energy sold at SRAC to the generator. This means that the generator would receive the benefits of the newily created RECs, which were not anticipated during the negotiation of the original PPAs. The SCE/PG\&E, and SMUD proposals assign all RECs sold under pre-restructuring long-term PPAs to the purchaser on behalf of ratepayers. The eventual disposition of RECs associated with renewable energy sold under pre-restructuring PPAs will have important implications on any negotiations that deal with restructuring or buying out PPAs.

One reason a renewables support program is being considered by the CPUC is the expectation that renewable power generators will have trouble competing in a competitive electricity market. The purpose of creating an REC market and REC procurement requirements for electric services providers is to provide the necessary increment of value (above market) necessary to allow renewables generators to produce renewable power in the restructured market. The economic viability of renewable generators that operate under PPAs, with energy sold under SRAC and long-term capacity sales, in the restructured market is questionable. Assuming SRAC represents full market value in the restructured market, as intended, facilities that receive SRAC plus capacity payments will be above market by the value of the capacity payments. How the value of capacity payments will compare with the value of the newly created RECs is difficult to predict.

The NCPA proposal addresses the issue of assigning RECs (in its case, RCCs) associated with renewable power sold under pre-restructuring PPAs by directing the parties to negotiate the disposition of the soon-to-be created RECs. The PPAs are legally binding contracts, and any changes to them will have to meet the requirements of contract law. The CPUC has posed as an important implementation issue the question of whether restructuring efforts will produce incentives to renegotiate contracts. The issue of assigning RECs under PPAs is one area where this issue must be considered carefully.

Competition andMarketing of RECs. The overall restructuring of the electricity market is predicated on the goal of making the market more competitive. The CPUC's renewables policy is intended to be subject to the rigors 
of market competition. Such competition can take a variety of forms. The broadest possible competition, which should lead to the lowest possible program cost (or maximum renewables production under the production credit program), would allow all renewables to compete together, among technologies, and between extant and new generating installations. Competition among renewables technologies has been discussed previously under the heading, Maintaining Renewables Diversity.

The restructuring policy goals for renewables include maintaining resource diversity and encouraging the development of new renewables. Developing new renewable generating sources may be difficult unless long-term contracts for sales of renewable energy and RECs can be obtained by developers who hope to secure funding for their projects. Most MRPR proposals leave the development of REC contracts to the market. No special provisions are included to facilitate the development of contracts tailored to the specific needs of new generating sources. The EDF et al. production credit proposal, in contrast, is for a program that would be tailored to developing new renewables, offering winning bidders 10 -year commitments to pay production credits, and barring current facilities from participating in the bidding program. IEP suggests enacting incentives to facilitate the development of new renewable generating sources. These include developing a renewable trademark easily recognized by consumers, offering a CTC credit option in which direct access customers who enter into contracts with renewable $\mathrm{QFs}$ would be eligible for a credit of all or a portion of the $\mathrm{CTC}$, and implementing a renewable energy purchase requirement for state facilities.

The CPUC decision relies on an enforceable standard to achieve its policy goals for renewables. It does not address the issue of green marketing directly. The Renewables Working Group, however, has asked each proposer to address the issue of how green marketing might fit into the proposals. The IEP proposal is designed around the concept of using green power marketing to achieve the bulk of the compliance that would be necessitated by the MRPR standard included as part of its proposal. Direct access providers will be able to qualify for green certification based on their acquiring enough RECs, which they will then be able to market as a desirable attribute of the service they offer to their customers. A rating system based on renewable content could be developed to provide consumers with a range of alternative green electrical services packages and prices.

Green power marketing is not a major ingredient of the other renewable program proposals, although two, renewables industries and SCE/PG\&E, discuss a mechanism by which green marketing techniques could be used to increase the generation of renewable energy. In each proposal, each electric services provider is obligated to acquire RECs that represent the MRPR fraction of its energy supply. Green marketing could be used by environmental organizations, for example, to competitively purchase and remove RECs from the system, which would increase the total quantity of renewable energy generated to a higher level than necessary to fulfill the collective mandated program obligation. Green direct access providers who purchase some multiplier greater than the MRPR standard of RECs for their portfolio of sources would have the same effect on the collective state market.

\section{Areas of Commonality and Difference among the Proposals}

Early in the process, the Renewables Working Group participants realized that a consensus on all or most major issues raised within the group in the timeframe envisioned would be unrealistic. The group recognized a wide diversity of interests among the parties, and disagreement over the methodology that should be used to implement a program to support renewable energy projects in California. The group decided to focus its efforts on developing a report that would present a number of comprehensive proposals for implementing the CPUC's renewables policy, and discuss the many issues that need to be resolved.

There is no unanimity of opinion on any major issue considered by the group, but some important areas of broad consensus, as well as areas of general disagreement, are highlighted below. 
The Renewables Working Group reached consensus in the following areas:

- Any renewables support program should rely, to the maximum extent possible, on market competition to minimize program cost or maximize program performance, or both. Incentives that encourage renewables to participate in the competitive market to the fullest extent possible should be developed.

- The program should be designed with maximum flexibility to facilitate compliance.

- Among the MRPR proposals there was agreement that a system of tradable credits should be established. However, the group did not agree about whether to denominate renewable credits using energy or capacity units, or at what level to set the initial level of the MRPR standard.

- Any renewables support program should be implemented statewide and be non-bypassable. However, there is disagreement as to whether that can be accomplished within the timeframe envisioned by the commission for initiating electric utility restructuring.

- To be eligible to participate in the program, energy produced by renewable generating sources must be used by California consumers. However, there is disagreement as to whether renewable generating sources outside California should be allowed to participate in the program (or whether they can be denied the right to participate).

- All MRPR proposals place compliance obligations on electrical services providers to meet the program's requirements. None place compliance obligations on electricity generators.

- All renewable generating technologies listed explicitly in the CPUC, including biomass, geothermal, solar, and wind, should be eligible to participate in a renewables support program. However, there is disagreement as to whether hydroelectric generators should be eligible to participate.

- Regardless of the type of renewables support program adopted, provisions should be included to counter any fraudulent activity.

- Coordinating with the $R D \& D$ working group regarding funding and other issues relating to the commercialization of emerging renewable generating technologies would be desirable.

The group was not able to reach consensus in the following areas:

- The basic methodology on which to base a renewables support program. There was disagreement about whether to base the program on an MRPR or on a surcharge funding mechanism.

- Whether the program should have a cost cap, at what level a cost cap should be set, and whether funds collected as a result of administrative sales of credits at the cap price should be turned back into the tradable-credit market or be administered in other ways.

- Whether the program should focus on developing new renewable generating sources, used to support current and new renewable generating sources. If current sources are eligible to participate in the program, there is disagreement over how to allocate credits for renewable energy that is sold under prerestructuring PPAs.

- Whether specific technologies should be targeted for support, or whether all renewables eligible to participate should compete head-to-head. The only technologies for which special consideration is re- 
quested are solid-fuel biomass, biogas, and emerging technologies. Some proposals would give special consideration to one or more of these technologies; others propose full head-to-head competition among all eligible renewable generating technologies. In the case of emerging technologies, the group was split over the role a renewables program should play in support of commercialization, or whether commercialization is better dealt with through the RD\&D program, or a combination of both.

- What types of noncentral station renewable energy applications should be eligible to participate? There was no consensus on whether UDCs can own distributed renewable generators, or whether gridconnected or off-grid self-generation, or both, should be eligible to participate.

- Can the CPUC implement a renewables program based on state law, or within the context of electric utility restructuring, or is new legislation required? For example, there was disagreement as to whether the commission could implement an MRPR proposal that imposes an obligation on retail providers other than UDCs.

- What is the best agency to administer the program, and what type of market structure should be used to trade and acquire renewable energy credits? Issues included governing the agency, identifying specific agency, role of the Power Exchange, ISO, and UDCs, and reliance on the private sector for developing a competitive REC market.

- There was disagreement as to whether the program should have a specified sunset date, or whether regular periodic reviews should be conducted. 


\section{Fiscal Tools and Technology Investments in Biomass Power}

The electric utility industry in the United States is on the verge of a radical makeover, as deregulation spreads across the country. Predicting the kinds of market opportunities that will become available for biomass power is difficult, but most experts believe that biomass generating sources will have trouble competing in a competitive marketplace from which environmental and diversity considerations are absent. Even in today's regulated market environment the biomass power industry is barely treading water. There is virtually no new project development activity in progress anywhere in the country, and no realistic prospect of a significant pickup. In the meantime, the industry has taken some significant losses during the past couple of years, as facilities throughout the country have accepted buyout agreements and shut down operations. More than $25 \%$ of California's operating biomass power generating capacity has disappeared since 1993, and many operating facilities are nearing the 11-year operating cliff in their PPAs when their power sales price reverts to the current short-run avoided cost $(\mathrm{SRAC})(\sim \$ 0.03 / \mathrm{kWh})$ from the fixed-price schedule $(>\$ 0.12 / \mathrm{kWh}$ in 1996$)$.

The rationale for providing public support to renewables in general, and to biomass in particular, is based on the desirable environmental, rural development, and fuel-diversity services provided by these generating sources. These services are discussed in detail in the companion volume to this report (Morris 1997). Public support for biomass and renewable power generation can be provided in a variety of ways, and can be instituted at the federal, state, and local jurisdictional levels. Identifying and supporting the most cost-effective options to maintain nonprice considerations in the marketplace is important for the biomass industry.

The modern biomass power industry in the United States was developed in response to a set of policy incentives that were offered beginning with the PURPA act of 1978. At the federal level, in addition to the PURPA legislation that created a market for electricity produced by nonutility generators, biomass power developers were offered significant financial incentives in the forms of tax credits and accelerated depreciation allowances. Some state and local jurisdictions added additional incentives for biomass energy development. For example, California offered tax credits and liberal accelerated depreciation at the state level for biomass power investments. The CPUC approved PPAs for biomass power generators that included a 10-year fixed-price schedule for power purchase rates that have proven to be far above market. Bond authorities issued low-interest, tax-free bonds that contributed to the financing of many biomass power plants.

The losses that have already occurred in the U.S. biomass power industry jeopardize not only the present operation of the industry, but the prospects for its future development. This is a result of two major factors. First, the shutdown of modern facilities after only about one-third of their originally projected operating lifetimes is a very bad signal to the financial community, which is the ultimate source of capital for industry development. Project finance is offered on very tight margins, and only to projects deemed to be reliable, long-term operating properties. Second, the significant development during the past 15 years in the U.S. biomass power industry has included the development of a considerable amount of expertise and infrastructure for producing biomass fuels from residue resources. When the industry contracts, demand for fuel decreases, and many residue generators find themselves in a situation in which they lose the fuel market, and have to find alternatives for disposing of their residue material. Attracting these sources back into the energy market will be more difficult because of the memory of the bad experiences that accompany the loss of biomass power generating capacity today.

In considering possible policy options for the future of the industry, we must understand the economic needs of biomass power generation. Conventional biomass power plants in the United States are based on steam turbine technology. Because of the size range of biomass generating facilities, and the moisture content of biomass fuel, biomass power generators cannot achieve the same heat rates as utility fossil fuel generators. Modern biomass power generators average about $15,000 \mathrm{Btu} / \mathrm{kWh}$, which translates into a fuel-price contribution to the cost of electricity production of approximately $\$ 0.01 / \mathrm{kWh}$ for each $\$ 10 / \mathrm{bdt}$ of fuel cost. In other words, delivered fuel 
that costs $\$ 25 / \mathrm{bdt}$ contributes $\$ 0.025 / \mathrm{kWh}$ to the cost of electricity production. The most efficient biomass generators can do about $20 \%$ better than this, reducing the fuel-cost contribution to $\$ 0.02 / \mathrm{Wh}$ for $\$ 25 / \mathrm{bdt}$ fuel.

Nonfuel operating costs for biomass power generators in the United States average about $\$ 0.025 / \mathrm{kWh}$ (Morris 1994). This covers labor, supplies, parts, maintenance, services, regulatory, insurance, overhead, and administrative costs. It does not include costs related to capital, or any provision for profits.

The turnkey construction cost for a modern, emissions-controlled biomass generating facility that uses steam turbine technology is about $\$ 1,500-\$ 2,000 / \mathrm{kW}$ of capacity. Financing costs and development expenses increase the total capital cost to about $\$ 2,000-\$ 2,500 / \mathrm{kW}$ of capacity. This translates into a capital cost contribution to the cost of electricity production of about $\$ 0.03$ to $\$ 0.05 / \mathrm{kWh}$, including return on investors' capital.

Biomass power facilities that purchase their fuel in a competitive market have operating costs of about $\$ 0.035$ to $\$ 0.06 / \mathrm{kWh}$, depending on their efficiency characteristics and regional biomass fuel costs. Most biomass power generators in California rely for some or all of their fuel supply on market sources. When capital costs are figured in, the cost of power production in a new biomass power plant that purchases fuel is about $\$ 0.065$ to $\$ 0.11 / \mathrm{kWh}$. This cost includes all capital-related costs, including a reasonable return on investment. Table 3 summarizes the cost of electricity production in a new biomass power plant in the United States. This level of compensation will be necessary to motivate substantial new development of biomass power plants that use conventional steam turbine technology.

Table 3. Cost of Electricity Production from Biomass

$\begin{array}{ll}\text { Nonfuel O\&M } & \$ / \mathrm{kWh} \\ \text { Fuel } & \$ 0.022-\$ 0.028 \\ \text { Capital } & \$ 0.012-\$ 0.035 \\ & \$ 0.030-\$ 0.050\end{array}$

In the current U.S. electricity market few utilities are willing to pay more than $\$ 0.03 / \mathrm{kWh}$ for purchased power, and many substantially less. Current SRACs in California are less $\$ 0.03 / \mathrm{kWh}$, and no utilities offer capacity payments. During the next several years competitive markets for electricity generation will be established, and the prices for power sales that will be available to biomass generators is difficult to anticipate. In California, utilities currently report to the CPUC that their average cost of power generation is $\$ 0.06$ to $\$ 0.07 / \mathrm{kWh}$. On the other hand, to estimate their stranded costs, the same utilities project power exchange prices for power purchases in the restructured market will be of $\$ 0.025$ to $\$ 0.035 / \mathrm{kWh}$. If these estimates are correct, fully amortized biomass power facilities would require above-market support of about $\$ 0.015$ to $\$ 0.04 / \mathrm{kWh}$ to remain in operation, and new facilities would require about $\$ 0.035$ to $\$ 0.08 / \mathrm{kWh}$ in above-market support to motivate their development.

An interesting topic of debate within the CPUC Renewables Working Group was the issue of program longevity. Some parties favored a permanent renewables program with no sunset provision; others favored a program designed to provide support for a defined period of time, during which the targeted technologies would be expected to use the support to achieve a state of market competitiveness with conventional technologies. Fixedterm support is best for programs intended to advance the commercialization of new technology, such as, in the biomass field, advanced gasification/combined cycle generation. If the intention of the program is to provide 
compensation for the ancillary environmental services provided by biomass, there is no compelling basis for ending the program on a predetermined schedule.

Public policy support for biomass and renewables can be targeted in a variety of ways. Several policy options have been suggested for the future support of the biomass and renewable power industries in the United States. The major options include:

- Tax credits and accelerated depreciation for investments in biomass generation

- Tax credits for biomass energy production

- Electric surcharge-funded production credits for renewable power generators

- Renewable portfolio standards for electric services providers

- $\quad$ Purchases of renewable energy by public sector energy consumers

- Incentives and structures for direct access and green marketing of renewables

- Technology $R \& D$ support.

Investment tax credits and accelerated depreciation can be enacted at the federal or state level, and have been used successfully during the past 15 years to help promote biomass and renewable energy development. Vir- tually all biomass facilities built in California benefited from tax advantages available for investments in biomass generating equipment. The investment tax credits are no longer available, but biomass generating equipment continues to be eligible for 5-year depreciation on federal taxes, as well as on many state taxes, including those in California.

EPAct created a \$0.015/kWh renewable energy tax credit (IRC § 45) for power produced using "closed-loop" biomass, which is defined as biomass grown specifically for fuel production. The tax credit so far has not been claimed by any taxpayer, as the domestic biomass power industry is based entirely on the use of waste and residue forms of biomass. Closed-loop biomass would be a more expensive fuel source, and its use would eliminate the special environmental value of biomass energy production as a superior disposal alternative for a variety of waste materials. Eliminating this restriction from the $\S 45$ tax credit, and extending it to power produced from biomass generators, would contribute substantially to the ability of such generators to remain in operation for the long-term.

EPAct also provides for a $\$ 0.015 / \mathrm{kWh}$ renewable energy production incentive (REPI) for energy produced by biomass generating facilities owned by municipal utilities and other public entities for which tax credits do not apply. The REPI is not constrained by the closed-loop biomass requirement, so qualifying facilities may use all kinds of residue forms of biomass. Like the $\S 45$ tax credit, the REPI applies only to facilities built between 1992 and 1999. There are no extant or planned municipally owned biomass generating facilities in California. A handful of landfill gas generators, but no solid biomass fuel generators, have qualified for this credit. One drawback of the REPI is that, although provided for in EPAct, it requires an annual appropriation to be available to eligible claimants.

Electric surcharge-funded production credits for biomass and renewable energy production would work for the biomass industry similarly to the REPI, with two important differences. First, the source of funding for the program would be a surcharge on consumers' electricity bills, rather than a loss of federal tax collections. Second, the value of the production credits (cents/kWh) would not be fixed administratively. Their value would be 
determined through a competitive bidding process, which would be designed to maximize the amount of biomass and renewable energy purchased, given the amount of surcharge funding available for distribution. The recent California restructuring legislation, $A B$ 1890, adopts a surcharge-funded production credit program for renewables during the transition period to full competition.

The RPS, public-sector facilities mandated purchase requirement, and direct access/green marketing programs are all designed to provide a market niche for energy produced from biomass and renewables. In these types of programs the above-market costs of the targeted technologies are compensated by electricity customers, who pay more than they would if they were buying from only the lowest-cost power sources available. The RPS approach mandates a minimum purchase requirement for all customers; the public-sector facilities purchase requirement imposes the obligation for above-market purchases on public sector energy users, which means that the abovemarket costs are paid for with general tax funds. In the green marketing approach the market niche is provided by motivating a few electric customers to purchase an energy supply with a guaranteed minimum fraction of renewables in the mix, even if that means paying a premium price.

Biomass and renewable energy technology R\&D support has long been a mainstay of federal energy policy. New technology development in the biomass power generation field has the potential to bring down the cost of biomass power production, thus closing the gap between the cost of power production using fossil fuels and biomass. This would reduce or eliminate the amount of above-market support needed to make biomass power production a viable generating option.

As Figure 3 shows, the cost of electricity production from biomass has three components, each of which is a significant part of the total cost of biomass power generation. The largest part of the cost, nearly $50 \%$, is capital related. The other half is divided between fuel and nonfuel O\&M, with nonfuel O\&M higher than fuel cost in most situations, except for cases in which fuel prices are unusually high. This means that technological fixes that add a capital cost burden to a facility in exchange for increases in power generating efficiency, but have no effect on nonfuel O\&M, are unlikely to be cost effective. Moreover, financial institutions will not be eager to invest new capital in the generating stock as the industry faces such great uncertainty. Burning natural gas in conjunction with biomass at the facilities is also unlikely to help, as the increase in boiler efficiency is not compensated for by the increase in fuel cost. Natural gas costs approximately $\$ 2.00 / \mathrm{MMBtu}$; biomass costs approximately $\$ 1.20 / \mathrm{MMBtu}$.

The hope for the biomass power generating industry in the United States probably lies in cost shifting and marketing. Facilities in California generate power for costs (fuel and O\&M) at $\$ 0.035$ to $\$ 0.06 / \mathrm{kWh}$. This may be more than the restructured market price offered for power through the exchange, but it is not outside the realm of marketability if direct-access sales, green-priced sales, or locational transmission values can be realized. A credit of $\$ 0.015 / \mathrm{kWh}$, or successful implementation of other cost-shifting strategies currently under consideration, would help keep these facilities in operation. 
Figure 3

\section{Cost of Biomass Power Production}

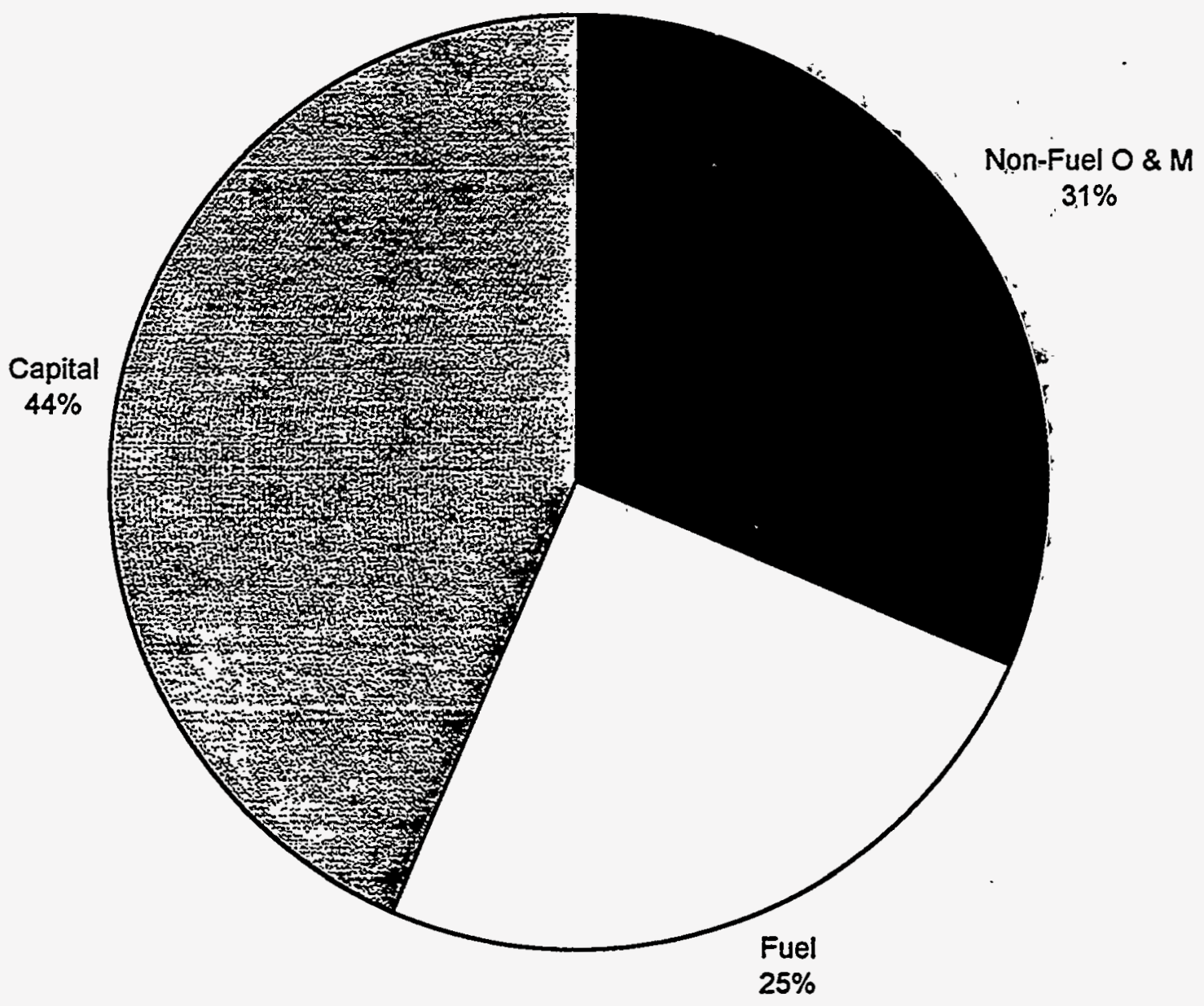




\section{References}

Morris, G. 1994. Fuel Use and Other Characteristics of Biomass Power Plants in the U.S. and Canada, FRA report, December.

Morris, G.; R. Wiser; and S. Pickle, eds. 1996. Renewables Working Group Report to the CPUC, CEC Report No. 500-96-008, August.

Morris, G. 1997. The Environmental Costs and Benefits of Biomass Energy Use in California, NREL report in publication, March. 


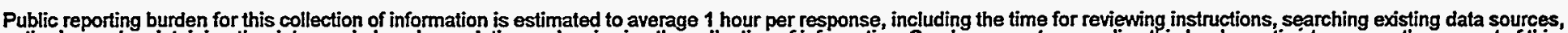

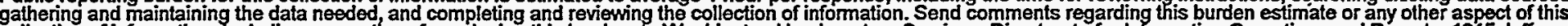

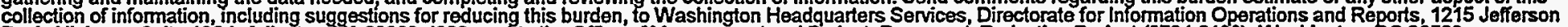

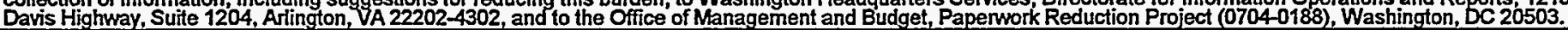
1. AGENCY USE ONLY (Leave blank)
2. REPORT DATE
3. REPORT TYPE AND DATES COVERED
May 1997
NREL Subcontract Report

\section{TITLE AND SUBTITLE}

Electricity Utility Restructuring and the California Biomass Energy Industry

5. FUNDING NUMBERS

Task\#: BP711010

6. AUTHOR(S)

G. Morris

\section{PERFORMING ORGANIZATION NAME(S) AND ADDRESS(ES)}

Future Resources Associates, Inc.

2039 Shattuck Ave., Suite 402

Berkeley. CA 94704

9. SPONSORING/MONITORING AGENCY NAME(S) AND ADDRESS(ES)

National Renewable Energy Laboratory

1617 Cole Boulevard

Golden, CO 80401-3393

\section{SUPPLEMENTARY NOTES}

\section{2a. DISTRIBUTION/AVAILABILITY STATEMENT}

National Technical Information Service

U.S. Department of Commerce

5285 Port Royal Road

Springfield, VA 22161

13. ABSTRACT (Maximum 200 words) A shock jolted the electric power industry in April 1994, when the Califomia Public Utilities Commission (CPUC) announced its intention to "restructure" the industry. The proposal, commonly referred to as retail wheeling, is based on the principle that market deregulation and competition will bring down the cost of electricity for all classes of customers. It would effectively break up the monopoly status of the regulated utilities and allow customers to purchase electricity directly from competing suppliers. According to the original CPUC proposal, cost alone would be the basis for determining which generating resources would be used. The proposal was modified in response to public inputs, and issued as a decision at the end of 1995 . The final proposal recognized the importance of renewables, and included provisions for a minimum renewables purchase requirement (MRPR). A Renewables Working Group convened to develop detailed proposals for implementing the CPUC's renewables program. Numerous proposals, which represented the range of possible programs that can be used to support renewables within the context of a restructured electric utility industry, were received.
14. SUBJECT TERMS
15. NUMBER OF PAGES 33

retail wheeling, biomass generating facilities, waste disposal, restructuring proposal
16. PRICE CODE

\section{SECURITY CLASSIFICATION OF REPORT}

\section{SECURITY CLASSIFICATION OF THIS PAGE}

\section{SECURITY CLASSIFICATION OF ABSTRACT}

20. LIMITATION OF ABSTRACT

Standard Form 298 (Rev. 2-89 Prescribed by ANSI Std. Z39-
NSN 7540-01-280-5500 